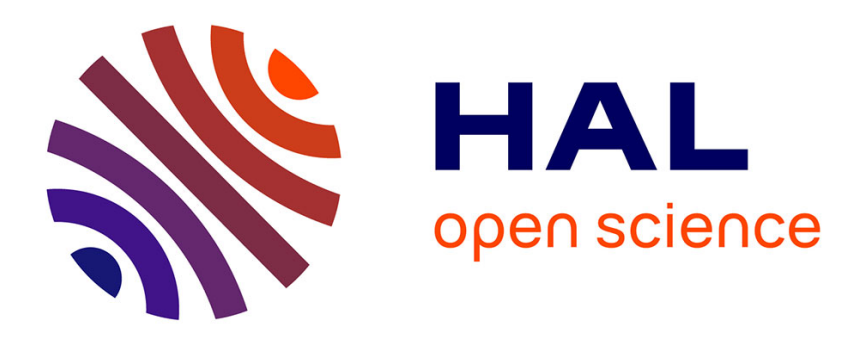

\title{
Hysteresis for ferromagnetism: asymptotics of some two-scale Landau-Lifshitz model
}

\author{
Eric Dumas, Stéphane Labbé
}

\section{To cite this version:}

Eric Dumas, Stéphane Labbé. Hysteresis for ferromagnetism: asymptotics of some two-scale LandauLifshitz model. Journal of Evolution Equations, 2012, 12 (3), pp.621-645. 10.1007/s00028-012-01471. hal-00625074

\section{HAL Id: hal-00625074 https://hal.science/hal-00625074}

Submitted on 20 Sep 2011

HAL is a multi-disciplinary open access archive for the deposit and dissemination of scientific research documents, whether they are published or not. The documents may come from teaching and research institutions in France or abroad, or from public or private research centers.
L'archive ouverte pluridisciplinaire HAL, est destinée au dépôt et à la diffusion de documents scientifiques de niveau recherche, publiés ou non, émanant des établissements d'enseignement et de recherche français ou étrangers, des laboratoires publics ou privés. 


\title{
Hysteresis for ferromagnetism: asymptotics of some 2-scale Landau-Lifshitz model
}

\author{
Eric Dumas*, Stéphane Labbé ${ }^{\dagger}$
}

September 20, 2011

\begin{abstract}
We study a 2-scale version of the Landau-Lifshitz system of ferromagnetism, introduced by Starynkevitch to modelize hysteresis: the response of the magnetization is fast compared to a slowly varying applied magnetic field. Taking the exchange term into account, in space dimension 3 , we prove that, under some natural stability assumption on the equilibria of the system, the strong solutions follow the dynamics of these equilibria. We also give explicit examples of relevant equilibria and exterior magnetic fields, when the ferromagnetic medium occupies some ellipsoidal domain.
\end{abstract}

\section{Contents}

1 Introduction 2

2 Statement of the results 3

3 Preliminaries $\quad 6$

3.1 Some functional analysis . . . . . . . . . . . . . . 6

3.2 About equilibria . . . . . . . . . . . . 8

4 Proof of Theorem 2.1 9

4.1 First step: the initial layer $\left[0, t_{\varepsilon}\right] \ldots \ldots \ldots \ldots \ldots$

4.1.1 Galerkine scheme . . . . . . . . . . . . . . . 10

$4.1 .2 \quad L^{2}$ estimates . . . . . . . . . . . . . . 11

$4.1 .3 \quad H^{2}$ estimates . . . . . . . . . . . . . 13

4.1 .4 Conclusion . . . . . . . . . . . . . . . . 18

4.1.5 Passing to the limit $k \rightarrow \infty \ldots \ldots \ldots \ldots$

4.2 Second step: following the slow dynamics after $t_{\varepsilon} \ldots \ldots 20$

*Université Grenoble 1, Institut Fourier, 100 rue des maths-BP 74, 38402 Saint Martin d'Hères cedex, France; edumas@ujf-grenoble.fr.

†Université Grenoble 1, Laboratoire Jean Kuntzmann, 51 rue des maths, 38402 Saint Martin d'Hères cedex, France; Stephane.Labbe@imag.fr. Support by The Nano-Science Foundation, Grenoble, Project HM-MAG is acknowledged. 
6 Appendix 23

6.1 About the dissipation property (2.5): proof of Lemmas 3.5 and 3.623

6.1.1 $L^{2}$ estimates . . . . . . . . . . . . . . 24

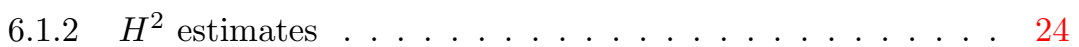

6.2 Proof of the commutator lemma $4.1 \ldots \ldots \ldots \ldots$

6.3 Proof of Gronwall's lemma $4.2 \ldots \ldots \ldots \ldots$

\section{Introduction}

Hysteresis is a widely studied, yet not completely understood phenomenon. It has played a role from the very beginning of the works on magnetism. Lord Rayleigh [9] proposed a model for ferromagnetic hysteresis in 1887, while the most achieved micromagnetism theory goes back to Landau and Lifshitz, in 1935 (see [7]).

In [12], Visintin gives many historical references, underlines the links between several forms of hysteresis (in particular, from plasticity, and from ferromagnetism), and how it is related to phase transitions. He performs a mathematical study of the so-called hysteresis operators, including the most famous one, due to Preisach.

Recently in [3], Carbou, Effendiev and Fabrie have proved the existence of strong solutions to a model of ferromagnetic hysteresis due to Effendiev.

In this paper, we rather investigate properties of a two-scale model introduced by Starynkevitch in [11]. This model describes the dynamics obtained when some exterior magnetic field is applied to the ferromagnetic material under consideration, while the response of the magnetization occurs on a much shorter time scale (say, denoted by $\varepsilon>0$ ). Mathematically, such models, associated to ordinary differential equations, had been studied in the nonstandard analysis framework, leading to "canard cycles" (see [6]). Considering a Landau-Lifshitz model in 0 space dimension (thus, an ODE), Starynkevitch studies the possible equilibria of the system, and the asymptotic behavior of the solutions (as the above mentioned parameter $\varepsilon$ goes to zero) when the exterior magnetic field slowly varies.

Our aim is to extend Starynkevitch's approach to the Landau-Lifshitz model in space dimension three, taking exchange term into account. This means, giving the asymptotic description of solutions to the slow-fast corresponding system of partial differential equations. Here, we prove such a result away from the bifurcation points of hysteresis loops. More precisely, assuming that the system (described by its magnetization) possesses at each time $t$ some stable equilibrium $m_{\mathrm{eq}}(t)$, and is submitted to some slowly varying exterior magnetic field, we show that the magnetization follows the dynamics of $m_{\text {eq }}$. We also give explicit examples (for ellipsoidal domains) of relevant equilibria and exterior magnetic fields. 


\section{Statement of the results}

The initial and boundary value problem associated to the 2 scale Landau-Lifshitz equation considered reads:

$$
\left\{\begin{array}{l}
\varepsilon \partial_{t} m^{\varepsilon}=m^{\varepsilon} \wedge h_{T}^{\varepsilon}-\alpha m^{\varepsilon} \wedge\left(m^{\varepsilon} \wedge h_{T}^{\varepsilon}\right), \text { for } t \geqslant 0, x \in \Omega, \\
\partial_{\nu} m_{\left.\right|_{\partial \Omega}}^{\varepsilon}=0, \\
m_{\left.\right|_{t=0} ^{\varepsilon}}^{\varepsilon}=m_{0} .
\end{array}\right.
$$

The unknown is the magnetization $m^{\varepsilon}$, function of the time variable $t \geqslant 0$ and of the space variable $x \in \Omega$, with values in the sphere $S^{2} \subset \mathbb{R}^{3}$. The domain $\Omega$ occupied by the ferromagnet is a subset of $\mathbb{R}^{3}$. Furthermore, $h_{T}^{\varepsilon}=h_{T}\left(t, m^{\varepsilon}(t)\right)$, where the total magnetic field $h_{T}$ is defined by

$$
h_{T}(t, m)=\overline{\Delta m}+h_{\mathrm{d}}(m)+h_{\text {ext }}(t) .
$$

Here, the first term $\Delta m$ is the "exchange term", which tends to impose a constant magnetization (domains where magnetization is constant are called "Weiss domains"), and $\overline{\Delta m}$ denotes the extension of $\Delta m$ by 0 out of $\Omega$. The second term, yielding spatial variations of the magnetization, is the "demagnetizing field" $h_{\mathrm{d}}(m)$, which results from a quasi-stationary approximation of Maxwell's equations; it is defined (at least, for $m \in L^{2}\left(\Omega, \mathbb{R}^{3}\right)$, as an element of $L^{2}\left(\mathbb{R}^{3}, \mathbb{R}^{3}\right)$ ) by

$$
\operatorname{curl} h_{\mathrm{d}}(m)=0 \quad \text { and } \quad \operatorname{div}\left(h_{\mathrm{d}}(m)+\bar{m}\right)=0 \quad \text { in } \mathbb{R}^{3} .
$$

Classical properties of the mapping $m \mapsto h_{\mathrm{d}}(m)$ are recalled in Section 3.1. The third term, $h_{\text {ext }}$, denotes some given exterior field, which is assumed to depend on time (and possibly on space). The positive constant $\alpha$ is some damping coefficient, which appears in the model when passing from a microscopic to a macroscopic description. The small parameter $\varepsilon>0$ expresses the fact that, while the exterior field $h_{\text {ext }}$ depends on $t$, and has time variations at scale 1 , the magnetization $m^{\varepsilon}$ essentially depends on $t / \varepsilon$, and thus has variations at the much more rapid scale $\varepsilon$.

Throughout this paper, for any $s \in \mathbb{N}$, we denote by $H^{s}(\Omega)$ the usual Sobolev space of functions with values in some vector space $\mathbb{R}^{N}$, whereas $H^{s}\left(\Omega, S^{2}\right)$ is the Sobolev space of functions with values in the sphere $S^{2}$ (which is not a vector space),

$$
H^{s}\left(\Omega, S^{2}\right)=\left\{m \in H^{s}(\Omega)|| m \mid \equiv 1 \text { almost everywhere }\right\} .
$$

Finally, for $s \geqslant 2, H_{N}^{s}(\Omega)$ denotes the subspace of functions in $H^{s}(\Omega)$ with homogeneous von Neumann boundary condition,

$$
H_{N}^{s}(\Omega)=\left\{m \in H^{s}(\Omega) \mid \partial_{\nu} m_{\mid \partial \Omega}=0\right\},
$$

and

$$
H_{N}^{s}\left(\Omega, S^{2}\right)=H^{s}\left(\Omega, S^{2}\right) \cap H_{N}^{s}(\Omega) .
$$

All these spaces (even if not vector spaces) inherit the (metric) topology given by the usual norm on $H^{s}(\Omega)$.

We prove the following 
Theorem 2.1. Let $\Omega$ be an open and bounded subset of $\mathbb{R}^{3}$, with smooth boundary. Let $T>0$, and $h_{\mathrm{ext}} \in C^{1}\left([0, T], C^{\infty}\left(\mathbb{R}^{3}\right)\right)$, bounded with bounded derivatives. Assume that there exist $m_{\mathrm{eq}} \in C^{1}\left([0, T], H_{N}^{2}\left(\Omega, S^{2}\right)\right)$ and $m_{0} \in H_{N}^{2}\left(\Omega, S^{2}\right)$ such that

(i) for all $t_{0} \in[0, T], m_{\mathrm{eq}}\left(t_{0}\right)$ is an equilibrium for

$$
\partial_{t} m=m \wedge h_{T}\left(t_{0}, m\right)-\alpha m \wedge\left(m \wedge h_{T}\left(t_{0}, m\right)\right)
$$

(see (3.1));

(ii) the solution $n_{0}$ to the initial and boundary value problem

$$
\left\{\begin{array}{l}
\partial_{t} n_{0}=n_{0} \wedge h_{T}\left(0, n_{0}\right)-\alpha n_{0} \wedge\left(n_{0} \wedge h_{T}\left(0, n_{0}\right)\right), \\
\partial_{\nu} n_{\left.0\right|_{\partial \Omega}}=0, \\
n_{\left.0\right|_{t=0}}=m_{0},
\end{array}\right.
$$

is global $\left(n_{0} \in C\left([0, \infty), H_{N}^{2}\left(\Omega, S^{2}\right)\right)\right)$, with $\nabla \Delta n_{0} \in L^{2}((0, \infty) \times \Omega)$, and $n_{0}(t)$ converges in $H^{2}(\Omega)$, as $t$ goes to $\infty$, towards $m_{\mathrm{eq}}(0)$;

(iii) the linearized operator $\mathcal{L}\left(\mathrm{m}_{\mathrm{eq}}\right)$ given by (4.31) has the following dissipation property:

$$
\begin{aligned}
& \text { there exist } C_{\mathrm{lin}}>0 \text { and } \eta>0 \text { such that, } \\
& \text { for all } \delta \in C\left([0, T], H^{\infty}(\Omega)\right) \text { with }\left|m_{\mathrm{eq}}+\delta\right| \equiv 1 \text { and } \partial_{\nu} \delta_{\mid \partial \Omega}=\partial_{\nu} \Delta \delta_{\mid \partial \Omega}=0, \\
& \sup _{t \in[0, T]}\|\delta(t)\|_{H^{2}(\Omega)} \leqslant \eta \text { implies: } \\
& \forall t \in[0, T], \quad\left(\mathcal{L}\left(\mathrm{t}, \mathrm{m}_{\mathrm{eq}}(\mathrm{t})\right) \delta(t) \mid \delta(t)\right)_{H^{2}(\Omega)} \leqslant-C_{\operatorname{lin}}\|\delta(t)\|_{H^{2}(\Omega)}^{2} .
\end{aligned}
$$

Then, there is $\varepsilon_{0}>0$ such that, for all $\varepsilon \in\left(0, \varepsilon_{0}\right)$, the solution $m^{\varepsilon}$ to (2.1) exists up to time $T\left(m^{\varepsilon} \in C\left([0, T], H_{N}^{2}\left(\Omega, S^{2}\right)\right)\right.$ ), and converges in $L^{2}\left((0, T), H^{2}(\Omega)\right) \cap$ $C\left([t, T], H^{2}(\Omega)\right)$ towards $m_{\mathrm{eq}}$ as $\varepsilon$ goes to zero, for all $t \in(0, T)$.

To prove Theorem 2.1, we first show that $m^{\varepsilon}$ converges to $m_{\mathrm{eq}}(0)$ within an initial layer of size $t_{\varepsilon}=C \varepsilon \ln (1 / \varepsilon)$. This is achieved via classical energy estimates (in $H^{2}$ ), carefully controlling the dependence upon $\varepsilon$-more technically speaking, the quasilinear and elliptic degenerate system of PDE's in (2.1) is first converted into a perturbation of some linear, strongly elliptic system, yielding the usual smooting properties, and a Galerkine approximation is used. In a second step, we prove that $m^{\varepsilon}$ converges towards $m_{\text {eq }}$ on the whole time interval $\left[t_{\varepsilon}, T\right]$. This amount to proving of long-time existence and return to equilibrium result for small initial data. Toward this end, we use again energy estimates, together with the stability assumption (2.5).

Figure 1 illustrates this corresponding asymptotic behaviour.

The above assumptions on the equilibrium $m_{\text {eq }}$ are discussed in Section 3.2 below. In particular, Assumption (ii) in Theorem 2.1 may be understood as a choice of 'prepared' data $m_{0}$ allowing to deal with the initial layer $(0, c \varepsilon \ln (1 / \varepsilon))$. 


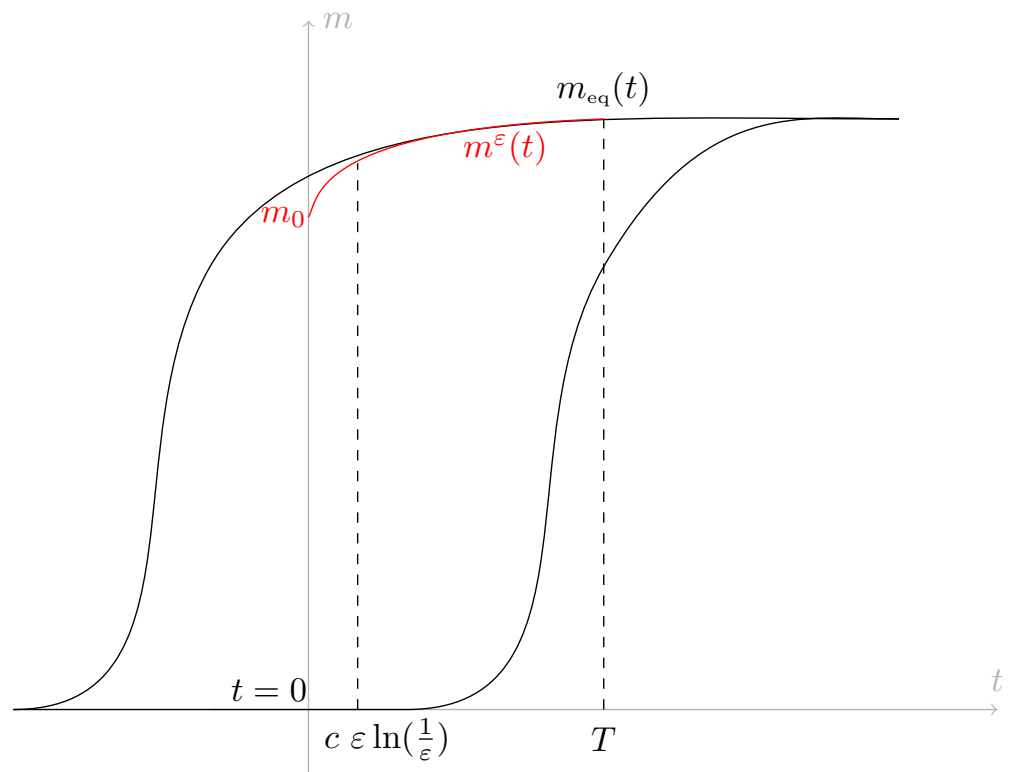

Figure 1: Dynamics of the magnetization away from bifurcation points.

Figure 1: Dynamics of the magnetization away from bifurcation points.

The dissipation property (2.5) expresses, for all $t_{0} \in[0, T]$, the stability of the linearization around $m_{\mathrm{eq}}\left(t_{0}\right)$ of (2.1), with $\varepsilon=1$ and with $h_{\mathrm{ext}}$ replaced with $h_{\text {ext }}\left(t_{0}\right)$, independent of time. This is a strong assumption, which ensures global existence of the solutions to the corresponding Landau-Lifshitz equation, for initial data close to $m_{\text {eq }}\left(t_{0}\right)$ :

Proposition 2.2. Let $\Omega$ be an open and bounded subset of $\mathbb{R}^{3}$, with smooth boundary. Consider an exterior magnetic field $h_{\mathrm{ext}} \in C^{\infty}\left(\mathbb{R}^{3}\right)$ (independent of time) bounded with bounded derivatives. Assume that there exists $m_{\mathrm{eq}} \in$ $H_{N}^{2}\left(\Omega, S^{2}\right)$ (independent of time) satisfying the equilibrium condition

$$
m_{\mathrm{eq}} \wedge\left(\Delta m_{\mathrm{eq}}+h_{\mathrm{d}}\left(m_{\mathrm{eq}}\right)+h_{\mathrm{ext}}\right)=0 \quad \text { on } \Omega,
$$

as well as the stability condition

there exist $C_{\text {lin }}>0$ and $\eta>0$ such that,

for all $\delta \in H^{\infty}(\Omega)$ with $\left|m_{\mathrm{eq}}+\delta\right| \equiv 1$ and $\partial_{\nu} \delta_{\left.\right|_{\partial \Omega}}=\partial_{\nu} \Delta \delta_{\left.\right|_{\partial \Omega}}=0$,

$\|\delta(t)\|_{H^{2}(\Omega)} \leqslant \eta$ implies:

$\left(\mathcal{L}\left(0, m_{\mathrm{eq}}\right) \delta \mid \delta\right)_{H^{2}(\Omega)} \leqslant-C_{\operatorname{lin}}\|\delta\|_{H^{2}(\Omega)}^{2}$, 
for the linearized operator $\mathcal{L}\left(0, m_{\mathrm{eq}}\right)$ given by $(4.31)$ (with $m_{\mathrm{eq}}(0)$ and $h_{\mathrm{ext}}(0)$ replaced with $m_{\mathrm{eq}}$ and $h_{\mathrm{ext}}$, respectively).

Then, there exists $\eta_{0}>0$ such that, for all $m_{0} \in H_{N}^{2}\left(\Omega, S^{2}\right)$ satisfying

$$
\left\|m_{0}-m_{\mathrm{eq}}\right\|_{H^{2}(\Omega)} \leqslant \eta_{0},
$$

the solution $n$ to the initial and boundary value problem

$$
\left\{\begin{array}{l}
\partial_{t} n=n \wedge h_{T}(0, n)-\alpha n \wedge\left(n \wedge h_{T}(0, n)\right), \\
\partial_{\nu} n_{\left.\right|_{\partial \Omega}}=0, \\
n_{\left.\right|_{t=0}}=m_{0},
\end{array}\right.
$$

is global $\left(n \in C\left([0, \infty), H_{N}^{2}\left(\Omega, S^{2}\right)\right)\right)$, with $\nabla \Delta\left(n-m_{\mathrm{eq}}\right) \in L^{2}((0, \infty) \times \Omega)$, and $n(t)$ converges in $H^{2}(\Omega)$, as $t$ goes to $\infty$, towards $m_{\mathrm{eq}}$.

In the case of $m_{\text {eq }}(0)$ constant over $\Omega$, Proposition 2.2 expresses that in Theorem 2.1, assumptions (i) and (iii) imply assumption (ii), so that we get:

Corollary 2.3. Let $\Omega$ be an open and bounded subset of $\mathbb{R}^{3}$, with smooth boundary. Let $T>0$, and $h_{\mathrm{ext}} \in C^{1}\left([0, T], C^{\infty}\left(\mathbb{R}^{3}\right)\right)$, bounded with bounded derivatives. Assume that there exist $m_{\mathrm{eq}} \in C^{1}\left([0, T], H_{N}^{2}\left(\Omega, S^{2}\right)\right)$ satisfying assumptions (i) and (iii) from Theorem 2.1. Assume furthermore that $m_{\mathrm{eq}}(0)$ is constant over $\Omega$.

Then, there exist $\eta_{0}, \varepsilon_{0}>0$ such that, for all $m_{0} \in H_{N}^{2}\left(\Omega, S^{2}\right)$ such that

$$
\left\|m_{0}-m_{\mathrm{eq}}(0)\right\|_{H^{2}(\Omega)} \leqslant \eta_{0},
$$

and for all $\varepsilon \in\left(0, \varepsilon_{0}\right)$, the solution $m^{\varepsilon}$ to (2.1) exists up to time $T$ ( $m^{\varepsilon} \in$ $\left.C\left([0, T], H_{N}^{2}\left(\Omega, S^{2}\right)\right)\right)$, and converges in $L^{2}\left((0, T), H^{2}(\Omega)\right) \cap C\left([t, T], H^{2}(\Omega)\right)$ towards $m_{\mathrm{eq}}$ as $\varepsilon$ goes to zero, for all $t \in(0, T)$.

In Lemma 3.5 below, we give examples (in ellipsoidal domains) of equilibria $m_{\text {eq }}$ satisfying the assumptions of Corollary 2.3.

\section{Preliminaries}

\subsection{Some functional analysis}

In this section, we recall some functional analysis results useful in the sequel. The first of them deals with the continuity properties of the demagnetizating field operator $h_{\mathrm{d}}$, immediately deduced from the Fourier representation $\widehat{h_{\mathrm{d}}(u)}(\xi)=-(\xi \cdot \hat{\bar{u}}(\xi)) \frac{\xi}{|\xi|^{2}}$ :

Lemma 3.1 ( $h_{d}$ properties). Let $\Omega$ be an open subset of $\mathbb{R}^{3}$. For all $s$ in $\mathbb{N}$ and $u$ in $H^{s}(\Omega)$, one has

$$
\left\|h_{\mathrm{d}}(u)\right\|_{H^{s}\left(\mathbb{R}^{3}\right)} \leqslant\|u\|_{H^{s}(\Omega)} .
$$


Furthermore, for all $v$ in $L^{2}(\Omega)$ we have

$$
\left(h_{\mathrm{d}}(u) \mid v\right)_{L^{2}(\Omega)}=-\left(u \mid h_{\mathrm{d}}(v)\right)_{L^{2}(\Omega)} .
$$

In addition to the usual Sobolev embeddings, we recall the following estimate, which results from the coercivity of the operator $A=1-\Delta$, with domain $D(A)=\left\{m \in H^{2}(\Omega) \mid \partial_{\nu} m_{\left.\right|_{\partial \Omega}}=0\right\}$ (see for example [5])

Lemma 3.2. Let $\Omega$ be a smooth bounded open set in $\mathbb{R}^{3}$. There exists a constant $C>0$ such that for all $u$ in $H_{N}^{2}(\Omega)$ one has

$$
\|u\|_{L^{\infty}(\Omega)} \leqslant C\left(\|u\|_{L^{2}(\Omega)}^{2}+\|\Delta u\|_{L^{2}(\Omega)}^{2}\right)^{\frac{1}{2}} .
$$

In the sequel, we will need the following definition.

Definition 3.3. Let $\Omega$ be a smooth bounded open set in $\mathbb{R}^{3}$. For $k \in \mathbb{N}^{\star}$, let $P_{k}$ be the $L^{2}(\Omega)$-orthogonal projection onto $V_{k}$, the vector space spanned by the first $k$ eigenfunctions of $A=1-\Delta$, with domain $D(A)=\left\{m \in H^{2}(\Omega) \mid \partial_{\nu} m_{\mid \partial \Omega}=0\right\}$.

The family of operators $\left(P_{k}\right)_{k \in \mathbb{N}}$ satisfies useful properties:

Lemma 3.4. The following properties are true.

(i) $\forall k \in \mathbb{N}^{\star}, \forall u \in D(A), \Delta P_{k} u=P_{k} \Delta u$,

(ii) $\forall k \in \mathbb{N}^{\star}, \forall s \in \mathbb{N}, \forall u \in H^{s}(\Omega), P_{k} u \in H^{s}(\Omega)$

(and $P_{k} u \in H_{N}^{s}(\Omega)$ when $s \geqslant 2$ ),

(iii) $\forall s \in \mathbb{N}, \lim _{k \rightarrow \infty}\left\|\left(1-P_{k}\right) u\right\|_{H^{s}(\Omega)}=0$ for all $u \in H^{s}(\Omega)$ when $s=0,1$, and for all $u \in H_{N}^{s}(\Omega)$ when $s \geqslant 2$.

Proof. (i) For all $u$ in $D(A), k$ in $\mathbb{N}^{\star}$, one has

$$
P_{k} \Delta u=\sum_{j=1}^{k}\left(\Delta u \mid \psi_{j}\right)_{L^{2}} \psi_{j},
$$

with $\left(\psi_{i}\right)_{i \in \mathbb{N}}$ the $L^{2}$-orthonormal basis of the eigenvectors of $\Delta$ associated to the eigenvalues $\left(\lambda_{i}\right)_{i \in \mathbb{N}}$. Then, using the vanishing Neumann boundary conditions,

$$
\left(\Delta u \mid \psi_{j}\right)_{L^{2}}=-\int_{\Omega} \nabla u \cdot \nabla \psi_{j}=\left(u \mid \Delta \psi_{j}\right)_{L^{2}}
$$

so that

$$
P_{k} \Delta u=\sum_{j=1}^{k} \lambda_{j}\left(u \mid \psi_{j}\right)_{L^{2}} \psi_{j}=\Delta P_{k} u .
$$

Point (ii) follows from the regularity properties of the family $\left(\psi_{i}\right)_{i \in \mathbb{N}}$.

Point (iii) is a consequence of the fact that $u \mapsto\left(\sum_{j=1}^{\infty}\left(1+\lambda_{j}^{s}\right)\left|\left(u \mid \psi_{j}\right)_{L^{2}}\right|^{2}\right)^{1 / 2}$ provides a norm equivalent to the usual one on $H^{s}(\Omega)$. 


\subsection{About equilibria}

Global solutions and equilibria. In [1, Th. 4.3], in the case of ellipsoidal domains $\Omega \subset \mathbb{R}^{3}$ and under a smallness assumption (on $\left\|h_{\text {ext }}\right\|_{L^{\infty}}$ and $\left.\left\|\Delta m_{0}\right\|_{L^{2}}\right)$, Alouges and Beauchard construct global smooth solutions to (2.1). Furthermore, these solutions satisfy

$$
\forall T>0, \quad\|\Delta m(T)\|_{L^{2}(\Omega)}^{2}+C \int_{0}^{T}\|\nabla \Delta m\|_{L^{2}(\Omega)}^{2} \leqslant\left\|\Delta m_{0}\right\|_{L^{2}(\Omega)},
$$

so that $\nabla \Delta m$ belongs to $L^{2}((0, \infty) \times \Omega)$. This is a part of our assumptions on the equilibrium $m_{\text {eq }}$, when requiring the existence of the global solution $n_{0}$. Saying that $m_{\text {eq }}\left(t_{0}\right)$ is an equilibrium for (2.3) means

$$
\left\{\begin{array}{l}
m_{\mathrm{eq}}\left(t_{0}\right) \wedge h_{T}\left(t_{0}, m_{\mathrm{eq}}\left(t_{0}\right)\right)=0 \\
\partial_{\nu} m_{\mathrm{eq}}\left(t_{0}\right)_{\mid \partial \Omega}=0
\end{array}\right.
$$

and requiring $H^{2}$ convergence of $n_{0}(t)$ towards $m_{\mathrm{eq}}(0)$ as $t$ goes to $\infty$ implies that $m_{\text {eq }}(0)$ is an equilibrium for $(2.3)$ with $t_{0}=0$.

Energy minimization. It is worth noting that energy decay occurs along the evolution of $n_{0}(t)$, so that one may hope at least $H^{1}$ convergence of $n_{0}(t)$ towards some local minimum of the energy, as $t$ goes to $\infty$. To the LandauLifshitz system (2.1) is associated the energy

$$
\mathcal{E}(t, m)=\frac{1}{2} \int_{\Omega}|\nabla m|^{2}-\frac{1}{2} \int_{\Omega} m \cdot h_{\mathrm{d}}(m)-\int_{\Omega} m \cdot h_{\mathrm{ext}}(t),
$$

and when $m$ is solution to (2.1), we have

$$
\frac{\mathrm{d}}{\mathrm{dt}} \mathcal{E}(t, m(t))=-\frac{\alpha}{\varepsilon}\left\|m(t) \wedge h_{T}(t, m(t))\right\|_{L^{2}(\Omega)}^{2}-\int_{\Omega} m(t) \cdot \partial_{t} h_{\mathrm{ext}}(t) .
$$

Since the exterior magnetic field does not depend on time during the evolution of $n_{0}$, we get

$$
\frac{\mathrm{d}}{\mathrm{dt}} \mathcal{E}\left(t, n_{0}(t)\right)=-\alpha\left\|n_{0}(t) \wedge h_{T}\left(t, n_{0}(t)\right)\right\|_{L^{2}(\Omega)}^{2} .
$$

In the case of ellipsoidal domains, special configurations are available. See [8], and references therein: there exists a real $3 \times 3$ definite positive diagonalizable matrix $D$ giving the demagnetizing field resulting from any magnetization constant constant over $\Omega$ :

$$
\forall v \in \mathbb{R}^{3}, \quad h_{\mathrm{d}}(v)_{\left.\right|_{\Omega}} \equiv-D v
$$

Hence, if $u \in S^{2}$ is an eigenvector of $D$ associated to the eigenvalue $d>0$, and if the exterior magnetic field is $h_{\mathrm{ext}}=\lambda u$ for some $\lambda>0\left(\right.$ or $h_{\mathrm{ext}}(x)=\lambda \chi(x) u$ 
for some $\chi \in C_{c}^{\infty}\left(\mathbb{R}^{3},[0,1]\right)$ to get a spatially localized field), then the system possesses two explicit equilibria $m_{\text {eq }}^{+}$and $m_{\text {eq }}^{-}$:

$$
m_{\mathrm{eq}}^{ \pm}= \pm u \text {. }
$$

One easily computes the energy associated to perturbations of these equilibria: for all $\delta \in H_{N}^{2}\left(\Omega, \mathbb{R}^{3}\right)$ such that $\left|m_{\text {eq }}^{ \pm}+\delta\right|=1$ a.e.,

$$
\mathcal{E}\left(m_{\mathrm{eq}}^{ \pm}+\delta\right)-\mathcal{E}\left(m_{\mathrm{eq}}^{ \pm}\right)=\frac{1}{2} \int_{\Omega}|\nabla \delta|^{2}-\frac{1}{2} \int_{\Omega} \delta \cdot h_{\mathrm{d}}(\delta)+\frac{1}{2}( \pm \lambda-d) \int_{\Omega}|\delta|^{2} .
$$

The first two terms are non-negative, so that for $\lambda$ large enough $(\lambda>d), m_{\text {eq }}^{+}$is a global minimum of $\mathcal{E}$; but for $\lambda$ small, it may fail to be even a local minimum. Concerning $m_{\mathrm{eq}}^{-}$, for all $\lambda>0$, if $d$ is the largest eigenvalue of $D$, and $\delta$ is constant in space, then the difference of energies above is less than $-\frac{\lambda}{2} \int_{\Omega}|\delta|^{2}$, thus negative, whereas for $\delta$ with large variations, the gradient term dominates, and the energy difference becomes positive. Hence, $m_{\mathrm{eq}}^{-}$is always a saddle point for $\mathcal{E}$.

The dissipation property (2.5). We have the following lemma, the proof of which is postponed to Section 6.1:

Lemma 3.5. For $\lambda>0$ large enough, the equilibrium $m_{\mathrm{eq}}^{+}$from (3.2) satisfies the dissipation property (2.5) (for some constant $C_{\text {lin }}$ depending on $\lambda$ ).

For $m_{\text {eq }}^{-}$, it is shown in Section 6.1 that for $\lambda$ large, we have on the contrary:

Lemma 3.6. For $\lambda>0$ large enough, there exist $C=C(\alpha, \lambda)>0$ and $\eta=\eta(\alpha, \lambda)>0$ such that, for all $\delta \in C\left([0, T], H^{\infty}(\Omega)\right)$ with $\left|m_{\mathrm{eq}}+\delta\right| \equiv$ 1 and $\partial_{\nu} \delta_{\left.\right|_{\partial \Omega}}=\partial_{\nu} \Delta \delta_{\left.\right|_{\partial \Omega}}=0$, when $\|\delta\|_{H^{2}(\Omega)} \leqslant \eta$, we have:

$$
\forall t \in[0, T], \quad\left(\mathcal{L}\left(t, m_{\mathrm{eq}}^{-}(t)\right) \delta(t) \mid \delta(t)\right)_{H^{2}(\Omega)} \geqslant C\|\delta\|_{H^{2}(\Omega)}^{2} .
$$

\section{Proof of Theorem 2.1}

First, consider the solution $n_{0}$ to the Cauchy problem

$$
\left\{\begin{array}{l}
\partial_{t} n_{0}=n_{0} \wedge h_{T}\left(0, n_{0}\right)-\alpha n_{0} \wedge\left(n_{0} \wedge h_{T}\left(0, n_{0}\right)\right), \\
\partial_{\nu} n_{\left.0\right|_{\partial \Omega}}=0 \\
n_{\left.0\right|_{t=0}}=m_{0}
\end{array}\right.
$$

and define $n^{\varepsilon}$ by

$$
\forall t \geqslant 0, \quad n^{\varepsilon}(t)=n_{0}(t / \varepsilon) .
$$

Then, $n^{\varepsilon} \in C_{b}\left([0, \infty), H^{2}(\Omega)\right)$ (with $\nabla \Delta n^{\varepsilon} \in L^{2}((0, \infty) \times \Omega)$, and we know that

$$
t_{\varepsilon} / \varepsilon \underset{\varepsilon \rightarrow 0}{\longrightarrow} \infty \quad \Longrightarrow \quad n^{\varepsilon}\left(t_{\varepsilon}\right) \underset{\varepsilon \rightarrow 0}{\longrightarrow} m_{\mathrm{eq}}(0) \text { in } H^{2}(\Omega) .
$$


Next, as in [4], we observe that for smooth functions $m$ with constant modulus (w.r.t. $x$ ), one has $m \cdot \Delta m=-2|\nabla m|^{2}$, so that smooth solutions to (2.1) equivalently satisfy

$$
\left\{\begin{array}{l}
\varepsilon \partial_{t} m^{\varepsilon}-\alpha \Delta m^{\varepsilon}=\mathcal{F}\left(t, m^{\varepsilon}\right), \\
\partial_{\nu} m_{\mid \partial \Omega}^{\varepsilon}=0, \\
m_{\left.\right|_{t=0} ^{\varepsilon}}^{\varepsilon}=m_{0},
\end{array}\right.
$$

where

$$
\mathcal{F}(t, m)=m \wedge h_{T}(t, m)+\alpha|\nabla m|^{2} m-\alpha m \wedge\left(m \wedge\left(h_{\mathrm{d}}(m)+h_{\mathrm{ext}}(t)\right)\right) .
$$

Furthermore, smooth $\left(L_{t}^{\infty} H_{x}^{2}\right)$ solutions to (4.2) issued from $m_{0}$ with constant modulus, equal to one, are shown to keep the same modulus for all time, (due to uniqueness of the solution $a=|m|^{2}$ to $\varepsilon \partial_{t} a=\alpha \Delta a+2 \alpha|\nabla u|^{2}(a-1), \partial_{\nu} a_{\left.\right|_{\partial \Omega}}=0$, $\left.a_{\left.\right|_{t=0}}=1\right)$. We thus solve (4.2) in the Banach space $C\left([0, T], H_{N}^{2}(\Omega)\right)$, and deduce from this conservation that the solution actually belongs to the space $C\left([0, T], H_{N}^{2}\left(\Omega, S^{2}\right)\right)$.

It is worth noting that (2.1) is an initial and boundary value problem for some quasilinear and parabolic degenerate operator, which is seen in (4.2) as a perturbation of a linear and strongly parabolic one.

Standard energy estimates ensure local-in-time existence and uniqueness of solutions continuous in time, with values in $H^{2}(\Omega)$ ) (with an existence time depending on $\varepsilon$ ): see for example [1] or [4]. By the usual continuation argument, we simply need to bound the $H^{2}$ norm of $m^{\varepsilon}$ to ensure existence up to time $T$. Actually, we shall prove convergence (as $\varepsilon$ goes to zero) at the same time, via energy estimates.

We first show that, after some time $t_{\varepsilon}$ of the form $t_{\varepsilon}=C \varepsilon \ln (1 / \varepsilon), m^{\varepsilon}$ and $n^{\varepsilon}$ are close: $\sup _{\left[0, t_{\varepsilon}\right]}\left\|m^{\varepsilon}-n^{\varepsilon}\right\|_{H^{2}(\Omega)}$ goes to zero with $\varepsilon$; thus, for $\varepsilon$ small enough, $m^{\varepsilon}\left(t_{\varepsilon}\right)$ is as close (in $H^{2}(\Omega)$ ) to $m_{\mathrm{eq}}(0)$ as desired. We then use the stability property of $m_{\mathrm{eq}}(t)$ to show that $m^{\varepsilon}(t)$ stays close to it, for $t \in\left[t_{\varepsilon}, T\right]$.

\subsection{First step: the initial layer $\left[0, t_{\varepsilon}\right]$}

\subsubsection{Galerkine scheme}

For $k \in \mathbb{N}^{\star}$, let $P_{k}$ be the $L^{2}(\Omega)$ - orthogonal projection onto $V_{k}$, the vector space spanned by the first $k$ eigenfunctions of $A=1-\Delta$, with domain $D(A)=\{m \in$ $\left.H^{2}(\Omega) \mid \partial_{\nu} m_{\left.\right|_{\partial \Omega}}=0\right\}$, as in Definition 3.3. Define a Galerkine approximation of (4.2) by:

$$
\left\{\begin{array}{l}
\varepsilon \partial_{t} m_{k}^{\varepsilon}-\alpha \Delta m_{k}^{\varepsilon}=P_{k} \mathcal{F}\left(t, m_{k}^{\varepsilon}\right) \\
m_{\left.k\right|_{t=0} ^{\varepsilon}}^{\varepsilon}=P_{k} m_{0}
\end{array}\right.
$$

The projection $n_{k}^{\varepsilon}=P_{k} n^{\varepsilon}$ also satisfies

$$
\begin{aligned}
\varepsilon \partial_{t} n_{k}^{\varepsilon}-\alpha \Delta n_{k}^{\varepsilon} & =P_{k} \mathcal{F}\left(0, n_{k}^{\varepsilon}\right)+\alpha\left[P_{k}, \Delta\right] n^{\varepsilon}+P_{k}\left(\mathcal{F}\left(0, n^{\varepsilon}\right)-\mathcal{F}\left(0, n_{k}^{\varepsilon}\right)\right) \\
& =P_{k} \mathcal{F}\left(0, n_{k}^{\varepsilon}\right)+P_{k}\left[P_{k}, \mathcal{F}(0, \cdot)\right]\left(n^{\varepsilon}\right),
\end{aligned}
$$


since for $u \in D(A), P_{k} \Delta u=\Delta P_{k} u$, according to Lemma 3.4.

Now, perform energy estimates (in $L^{2}$ ) for $\varphi_{k}^{\varepsilon}=m_{k}^{\varepsilon}-n_{k}^{\varepsilon}$, solution to

$$
\left\{\begin{array}{l}
\varepsilon \partial_{t} \varphi_{k}^{\varepsilon}-\alpha \Delta \varphi_{k}^{\varepsilon}=P_{k}\left(\mathcal{F}\left(t, m_{k}^{\varepsilon}\right)-\mathcal{F}\left(0, n_{k}^{\varepsilon}\right)\right)-P_{k}\left[P_{k}, \mathcal{F}(0, \cdot)\right]\left(n^{\varepsilon}\right), \\
\varphi_{\left.k\right|_{t=0} ^{\varepsilon}}^{\varepsilon}=0 .
\end{array}\right.
$$

\subsection{2 $\quad L^{2}$ estimates}

Take the scalar product (in $L^{2}(\Omega)$ ) of $\varphi_{k}^{\varepsilon}$ with the first equation in (4.5) to get

$$
\frac{\varepsilon}{2} \frac{\mathrm{d}}{\mathrm{dt}}\left(\left\|\varphi_{k}^{\varepsilon}\right\|_{L^{2}(\Omega)}^{2}\right)+\alpha\left\|\nabla \varphi_{k}^{\varepsilon}\right\|_{L^{2}(\Omega)}^{2}=I_{1}+I_{2}+I_{3}+I_{4},
$$

with

$$
\begin{aligned}
& I_{1}=\left(\varphi_{k}^{\varepsilon} \mid m_{k}^{\varepsilon} \wedge h_{T}\left(t, m_{k}^{\varepsilon}\right)-n_{k}^{\varepsilon} \wedge h_{T}\left(0, n_{k}^{\varepsilon}\right)\right)_{L^{2}(\Omega)}, \\
& I_{2}=\alpha\left(\left.\varphi_{k}^{\varepsilon}|| \nabla m_{k}^{\varepsilon}\right|^{2} m_{k}^{\varepsilon}-\left|\nabla n_{k}^{\varepsilon}\right|^{2} n_{k}^{\varepsilon}\right)_{L^{2}(\Omega)}, \\
& I_{3}=-\alpha\left(\varphi_{k}^{\varepsilon} \mid m_{k}^{\varepsilon} \wedge\left(m_{k}^{\varepsilon} \wedge\left(h_{\mathrm{d}}\left(m_{k}^{\varepsilon}\right)+h_{\mathrm{ext}}(t)\right)\right)\right. \\
& \left.-n_{k}^{\varepsilon} \wedge\left(n_{k}^{\varepsilon} \wedge\left(h_{\mathrm{d}}\left(n_{k}^{\varepsilon}\right)+h_{\mathrm{ext}}(0)\right)\right)\right)_{L^{2}(\Omega)}, \\
& I_{4}=\left(\varphi_{k}^{\varepsilon} \mid\left[P_{k}, \mathcal{F}(0, \cdot)\right]\left(n^{\varepsilon}\right)\right)_{L^{2}(\Omega)} .
\end{aligned}
$$

Estimating $I_{1}$. Decompose $m_{k}^{\varepsilon}=n_{k}^{\varepsilon}+\varphi_{k}^{\varepsilon}$. For all $\varphi, h \in \mathbb{R}^{3}, \varphi \cdot(\varphi \wedge h)=0$, so that

$$
\begin{aligned}
I_{1}=\left(\varphi_{k}^{\varepsilon} \mid n_{k}^{\varepsilon} \wedge\left(\Delta\left(n_{k}^{\varepsilon}+\varphi_{k}^{\varepsilon}\right)+h_{\mathrm{d}}\left(n_{k}^{\varepsilon}+\varphi_{k}^{\varepsilon}\right)+h_{\mathrm{ext}}(t)\right)\right. \\
\left.\quad-n_{k}^{\varepsilon} \wedge\left(\Delta n_{k}^{\varepsilon}+h_{\mathrm{d}}\left(n_{k}^{\varepsilon}\right)+h_{\mathrm{ext}}(0)\right)\right)_{L^{2}(\Omega)} \\
=\left(\varphi_{k}^{\varepsilon} \mid n_{k}^{\varepsilon} \wedge\left(\Delta \varphi_{k}^{\varepsilon}+h_{\mathrm{d}}\left(\varphi_{k}^{\varepsilon}\right)\right)\right)_{L^{2}(\Omega)}+\left(\varphi_{k}^{\varepsilon} \mid n_{k}^{\varepsilon} \wedge\left(h_{\mathrm{ext}}(t)-h_{\mathrm{ext}}(0)\right)\right)_{L^{2}(\Omega)} .
\end{aligned}
$$

Using the continuity of $h_{\mathrm{d}}$ on $L^{2}(\Omega)$, we get, for some constant $C$ depending on $\left\|\partial_{t} h_{\mathrm{ext}}\right\|_{L_{t, x}^{\infty}}$ and $\left\|n_{0}\right\|_{L^{\infty}\left((0, \infty), L^{2}(\Omega)\right)}$ :

$$
I_{1} \leqslant C\left\|\varphi_{k}^{\varepsilon}\right\|_{L^{2}(\Omega)}\left(\left\|\varphi_{k}^{\varepsilon}\right\|_{H^{2}(\Omega)}+t\right) .
$$

Estimating $I_{2}$. Write

$$
\begin{aligned}
\left|\nabla m_{k}^{\varepsilon}\right|^{2} m_{k}^{\varepsilon}-\left|\nabla n_{k}^{\varepsilon}\right|^{2} n_{k}^{\varepsilon} & =\left(\left|\nabla m_{k}^{\varepsilon}\right|^{2}-\left|\nabla n_{k}^{\varepsilon}\right|^{2}\right) m_{k}^{\varepsilon}+\left|\nabla n_{k}^{\varepsilon}\right|^{2} \varphi_{k}^{\varepsilon} \\
& =\left(\nabla\left(2 n_{k}^{\varepsilon}+\varphi_{k}^{\varepsilon}\right) \cdot \nabla \varphi_{k}^{\varepsilon}\right)\left(n_{k}^{\varepsilon}+\varphi_{k}^{\varepsilon}\right)+\left|\nabla n_{k}^{\varepsilon}\right|^{2} \varphi_{k}^{\varepsilon} .
\end{aligned}
$$

Then, use Sobolev's embeddings, such as

$$
\begin{aligned}
\left(\varphi_{k}^{\varepsilon} \mid\left(\nabla n_{k}^{\varepsilon} \cdot \nabla \varphi_{k}^{\varepsilon}\right) n_{k}^{\varepsilon}\right)_{L^{2}(\Omega)} & \leqslant\left\|\varphi_{k}^{\varepsilon}\right\|_{L^{\infty}(\Omega)}\left\|\nabla n_{k}^{\varepsilon}\right\|_{L^{2}(\Omega)}\left\|\nabla \varphi_{k}^{\varepsilon}\right\|_{L^{4}(\Omega)}\left\|n_{k}^{\varepsilon}\right\|_{L^{4}(\Omega)} \\
& \lesssim\left\|\varphi_{k}^{\varepsilon}\right\|_{H^{2}(\Omega)}\left\|n_{k}^{\varepsilon}\right\|_{H^{1}(\Omega)}\left\|\nabla \varphi_{k}^{\varepsilon}\right\|_{H^{1}(\Omega)}\left\|n_{k}^{\varepsilon}\right\|_{H^{1}(\Omega)},
\end{aligned}
$$


and

$$
\left(\left.\varphi_{k}^{\varepsilon}|| \nabla n_{k}^{\varepsilon}\right|^{2} \varphi_{k}^{\varepsilon}\right)_{L^{2}(\Omega)} \leqslant\left\|\varphi_{k}^{\varepsilon}\right\|_{L^{\infty}(\Omega)}^{2}\left\|\nabla n_{k}^{\varepsilon}\right\|_{L^{2}(\Omega)}^{2} \lesssim\left\|\varphi_{k}^{\varepsilon}\right\|_{H^{2}(\Omega)}^{2}\left\|n_{k}^{\varepsilon}\right\|_{H^{1}(\Omega)}^{2},
$$

to get the estimate

$$
I_{2} \leqslant C\left\|\varphi_{k}^{\varepsilon}\right\|_{H^{2}(\Omega)}\left(\left\|\varphi_{k}^{\varepsilon}\right\|_{H^{2}(\Omega)}+\left\|\varphi_{k}^{\varepsilon}\right\|_{H^{2}(\Omega)}^{3}\right),
$$

for some constant $C$ depending on $\left\|n_{0}\right\|_{L^{\infty}\left((0, \infty), H^{1}(\Omega)\right)}$.

Estimating $I_{3}$. As for $I_{1}$, cancellations allow to write

$$
\begin{aligned}
I_{3}=- & \alpha\left(\varphi_{k}^{\varepsilon} \mid n_{k}^{\varepsilon} \wedge\left(n_{k}^{\varepsilon} \wedge h_{\mathrm{d}}\left(\varphi_{k}^{\varepsilon}\right)+\varphi_{k}^{\varepsilon} \wedge h_{\mathrm{d}}\left(n_{k}^{\varepsilon}\right)\right)+n_{k}^{\varepsilon} \wedge\left(\varphi_{k}^{\varepsilon} \wedge h_{\mathrm{ext}}(t)\right)\right)_{L^{2}(\Omega)} \\
& -\alpha\left(\varphi_{k}^{\varepsilon} \mid n_{k}^{\varepsilon} \wedge\left(n_{k}^{\varepsilon} \wedge\left(h_{\mathrm{ext}}(t)-h_{\mathrm{ext}}(0)\right)\right)\right)_{L^{2}(\Omega)} .
\end{aligned}
$$

Boundedness of $h_{\mathrm{d}}$ on $L^{p}$ for finite $p$ provides the bounds

$$
\left\|n_{k}^{\varepsilon} \wedge\left(n_{k}^{\varepsilon} \wedge h_{\mathrm{d}}\left(\varphi_{k}^{\varepsilon}\right)\right)\right\|_{L^{2}(\Omega)} \leqslant\left\|n_{k}^{\varepsilon}\right\|_{L^{6}(\Omega)}^{2}\left\|\varphi_{k}^{\varepsilon}\right\|_{L^{6}(\Omega)},
$$

and

$$
\left\|n_{k}^{\varepsilon} \wedge\left(\varphi_{k}^{\varepsilon} \wedge h_{\mathrm{d}}\left(n_{k}^{\varepsilon}\right)\right)\right\|_{L^{2}(\Omega)} \leqslant\left\|n_{k}^{\varepsilon}\right\|_{L^{6}(\Omega)}^{2}\left\|\varphi_{k}^{\varepsilon}\right\|_{L^{6}(\Omega)} .
$$

The above $L^{6}$ norms are controlled by $H^{1}$ norms. Thus, for some constant $C$ depending on $\left\|h_{\text {ext }}\right\|_{L_{t, x}^{\infty}},\left\|\partial_{t} h_{\text {ext }}\right\|_{L_{t, x}^{\infty}}$ and $\left\|n_{0}\right\|_{L^{\infty}\left((0, \infty), H^{1}(\Omega)\right)}$ :

$$
I_{3} \leqslant C\left\|\varphi_{k}^{\varepsilon}\right\|_{L^{2}(\Omega)}\left(\left\|\varphi_{k}^{\varepsilon}\right\|_{H^{2}(\Omega)}+t\right) .
$$

Estimating $I_{4}$. Setting $r_{k}^{\varepsilon}=\left\|\left[P_{k}, \mathcal{F}(0, \cdot)\right]\left(n_{k}^{\varepsilon}\right)\right\|_{L^{2}(\Omega)}^{2}$, we have:

$$
I_{4} \leqslant\left\|\varphi_{k}^{\varepsilon}\right\|_{L^{2}(\Omega)}^{2}+r_{k}^{\varepsilon} \text {, and } r_{k}^{\varepsilon} \underset{k \rightarrow \infty}{\longrightarrow} 0 \text { in } L^{1}(0, T) \text { for all } T>0 \text {, with } \varepsilon \text { fixed. }
$$

This is a consequence of the following lemma, the proof of which is postponed to Section 6.2.

Lemma 4.1. For all $T>0$ and $n \in C\left([0, T], H_{N}^{2}(\Omega)\right) \cap L^{2}\left((0, T), H^{3}(\Omega)\right)$,

$$
\left[P_{k}, \mathcal{F}(0, \cdot)\right](n) \underset{k \rightarrow \infty}{\longrightarrow} 0 \text { in } L^{2}\left((0, T), H^{1}(\Omega)\right) .
$$

Gathering $L^{2}$ estimates. Adding (4.6), (4.7), (4.8) and (4.9), we get

$$
\begin{aligned}
\frac{\varepsilon}{2} \frac{\mathrm{d}}{\mathrm{dt}}\left(\left\|\varphi_{k}^{\varepsilon}\right\|_{L^{2}(\Omega)}^{2}\right) & +\alpha\left\|\nabla \varphi_{k}^{\varepsilon}\right\|_{L^{2}(\Omega)}^{2} \\
& \leqslant C\left\|\varphi_{k}^{\varepsilon}\right\|_{H^{2}(\Omega)}\left(t+\left\|\varphi_{k}^{\varepsilon}\right\|_{H^{2}(\Omega)}+\left\|\varphi_{k}^{\varepsilon}\right\|_{H^{2}(\Omega)}^{3}+r_{k}^{\varepsilon}\right),
\end{aligned}
$$

for some constant depending on the quantities $\left\|h_{\text {ext }}\right\|_{L_{t, x}^{\infty}},\left\|\partial_{t} h_{\text {ext }}\right\|_{L_{t, x}^{\infty}}$ and $\left\|n_{0}\right\|_{L^{\infty}\left((0, \infty), H^{1}(\Omega)\right)}$, and $r_{k}^{\varepsilon}$ from (4.9). 


\subsection{3 $H^{2}$ estimates}

Next, take the scalar product in $L^{2}(\Omega)$ of $\Delta^{2} \varphi_{k}^{\varepsilon}$ with the first equation in (4.5) to get

$$
\frac{\varepsilon}{2} \frac{\mathrm{d}}{\mathrm{dt}}\left(\left\|\Delta \varphi_{k}^{\varepsilon}\right\|_{L^{2}(\Omega)}^{2}\right)+\alpha\left\|\nabla \Delta \varphi_{k}^{\varepsilon}\right\|_{L^{2}(\Omega)}^{2}=I I_{1}+I I_{2}+I I_{3}+I I_{4},
$$

with

$$
\begin{aligned}
& I I_{1}=\left(\Delta^{2} \varphi_{k}^{\varepsilon} \mid m_{k}^{\varepsilon} \wedge h_{T}\left(t, m_{k}^{\varepsilon}\right)-n_{k}^{\varepsilon} \wedge h_{T}\left(0, n_{k}^{\varepsilon}\right)\right)_{L^{2}(\Omega)}, \\
& I I_{2}=\alpha\left(\left.\Delta^{2} \varphi_{k}^{\varepsilon}|| \nabla m_{k}^{\varepsilon}\right|^{2} m_{k}^{\varepsilon}-\left|\nabla n_{k}^{\varepsilon}\right|^{2} n_{k}^{\varepsilon}\right)_{L^{2}(\Omega)}, \\
& I I_{3}=-\alpha\left(\Delta^{2} \varphi_{k}^{\varepsilon} \mid m_{k}^{\varepsilon} \wedge\left(m_{k}^{\varepsilon} \wedge\left(h_{\mathrm{d}}\left(m_{k}^{\varepsilon}\right)+h_{\mathrm{ext}}(t)\right)\right)\right. \\
& \left.\quad-n_{k}^{\varepsilon} \wedge\left(n_{k}^{\varepsilon} \wedge\left(h_{\mathrm{d}}\left(n_{k}^{\varepsilon}\right)+h_{\mathrm{ext}}(0)\right)\right)\right)_{L^{2}(\Omega)}, \\
& I I_{4}=\left(\Delta^{2} \varphi_{k}^{\varepsilon} \mid\left[P_{k}, \mathcal{F}(0, \cdot)\right]\left(n^{\varepsilon}\right)\right)_{L^{2}(\Omega)} .
\end{aligned}
$$

Estimating $I I_{1}$. Split

$$
I I_{1}=I I_{1,1}+I I_{1,2}+I I_{1,3}
$$

with

$$
\begin{aligned}
& I I_{1,1}=\left(\Delta^{2} \varphi_{k}^{\varepsilon} \mid m_{k}^{\varepsilon} \wedge\left(\Delta \varphi_{k}^{\varepsilon}+h_{\mathrm{d}}\left(\varphi_{k}^{\varepsilon}\right)\right)\right)_{L^{2}(\Omega)}, \\
& I I_{1,2}=\left(\Delta^{2} \varphi_{k}^{\varepsilon} \mid \varphi_{k}^{\varepsilon} \wedge h_{T}\left(t, n_{k}^{\varepsilon}\right)\right)_{L^{2}(\Omega)}, \\
& I I_{1,3}=\left(\Delta^{2} \varphi_{k}^{\varepsilon} \mid n_{k}^{\varepsilon} \wedge\left(h_{\mathrm{ext}}(t)-h_{\mathrm{ext}}(0)\right)\right)_{L^{2}(\Omega)} .
\end{aligned}
$$

The first term is written

$$
I I_{1,1}=\left(\Delta^{2} \varphi_{k}^{\varepsilon} \mid n_{k}^{\varepsilon} \wedge\left(\Delta \varphi_{k}^{\varepsilon}+h_{\mathrm{d}}\left(\varphi_{k}^{\varepsilon}\right)\right)\right)_{L^{2}(\Omega)}+\left(\Delta^{2} \varphi_{k}^{\varepsilon} \mid \varphi_{k}^{\varepsilon} \wedge\left(\Delta \varphi_{k}^{\varepsilon}+h_{\mathrm{d}}\left(\varphi_{k}^{\varepsilon}\right)\right)\right)_{L^{2}(\Omega)} \text {. }
$$

Integrating by parts,

$$
\begin{aligned}
& \left(\Delta^{2} \varphi_{k}^{\varepsilon} \mid n_{k}^{\varepsilon} \wedge\left(\Delta \varphi_{k}^{\varepsilon}+h_{\mathrm{d}}\left(\varphi_{k}^{\varepsilon}\right)\right)\right)_{L^{2}(\Omega)}= \\
& -\left(\nabla \Delta \varphi_{k}^{\varepsilon} \mid \nabla n_{k}^{\varepsilon} \wedge\left(\Delta \varphi_{k}^{\varepsilon}+h_{\mathrm{d}}\left(\varphi_{k}^{\varepsilon}\right)\right)+n_{k}^{\varepsilon} \wedge \nabla h_{\mathrm{d}}\left(\varphi_{k}^{\varepsilon}\right)\right)_{L^{2}(\Omega)} \\
& \leqslant \eta\left\|\nabla \Delta \varphi_{k}^{\varepsilon}\right\|_{L^{2}(\Omega)}^{2}+\frac{1}{4 \eta}\left\|\nabla n_{k}^{\varepsilon} \wedge\left(\Delta \varphi_{k}^{\varepsilon}+h_{\mathrm{d}}\left(\varphi_{k}^{\varepsilon}\right)\right)+n_{k}^{\varepsilon} \wedge \nabla h_{\mathrm{d}}\left(\varphi_{k}^{\varepsilon}\right)\right\|_{L^{2}(\Omega)}^{2} \\
& \leqslant \eta\left\|\nabla \Delta \varphi_{k}^{\varepsilon}\right\|_{L^{2}(\Omega)}^{2}+C_{\eta}\left(\left\|n_{k}^{\varepsilon}\right\|_{H^{2}(\Omega)}^{2}+\left\|\nabla \Delta n_{k}^{\varepsilon}\right\|_{L^{2}(\Omega)}^{2}\right)\left\|\varphi_{k}^{\varepsilon}\right\|_{H^{2}(\Omega)},
\end{aligned}
$$


for all $\eta>0$, for some (large) constant $C_{\eta}$, using Sobolev's inequalities. ¿From this, we deduce that for all $\eta>0$, there exists $C_{\eta}>0$, depending only on $\left\|n_{0}\right\|_{L^{\infty}\left((0, \infty), H^{2}(\Omega)\right)}$, such that

$$
\begin{aligned}
&\left(\Delta^{2} \varphi_{k}^{\varepsilon} \mid n_{k}^{\varepsilon} \wedge\left(\Delta \varphi_{k}^{\varepsilon}+h_{\mathrm{d}}\left(\varphi_{k}^{\varepsilon}\right)\right)\right)_{L^{2}(\Omega)} \leqslant \\
& \eta\left\|\nabla \Delta \varphi_{k}^{\varepsilon}\right\|_{L^{2}(\Omega)}^{2}+C_{\eta}\left(1+\left\|\nabla \Delta n_{k}^{\varepsilon}\right\|_{L^{2}(\Omega)}^{2}\right)\left\|\varphi_{k}^{\varepsilon}\right\|_{H^{2}(\Omega)}^{2} .
\end{aligned}
$$

Integrating by parts again,

$$
\begin{aligned}
& \left(\Delta^{2} \varphi_{k}^{\varepsilon} \mid \varphi_{k}^{\varepsilon} \wedge\left(\Delta \varphi_{k}^{\varepsilon}+h_{\mathrm{d}}\left(\varphi_{k}^{\varepsilon}\right)\right)\right)_{L^{2}(\Omega)}= \\
& -\left(\nabla \Delta \varphi_{k}^{\varepsilon} \mid \nabla \varphi_{k}^{\varepsilon} \wedge\left(\Delta \varphi_{k}^{\varepsilon}+h_{\mathrm{d}}\left(\varphi_{k}^{\varepsilon}\right)\right)+\varphi_{k}^{\varepsilon} \wedge \nabla h_{\mathrm{d}}\left(\varphi_{k}^{\varepsilon}\right)\right)_{L^{2}(\Omega)} \\
& \leqslant\left\|\nabla \Delta \varphi_{k}^{\varepsilon}\right\|_{L^{2}(\Omega)}\left\|\nabla \varphi_{k}^{\varepsilon}\right\|_{L^{\infty}(\Omega)}\left\|\Delta \varphi_{k}^{\varepsilon}\right\|_{L^{2}(\Omega)} \\
& \left.\left.\quad+\left\|\nabla \Delta \varphi_{k}^{\varepsilon}\right\|_{L^{2}(\Omega)}\left(\left\|\nabla \varphi_{k}^{\varepsilon}\right\|_{L^{4}(\Omega)} \| h_{\mathrm{d}}\left(\varphi_{k}^{\varepsilon}\right)\right)\left\|_{L^{4}(\Omega)}+\right\| \varphi_{k}^{\varepsilon}\left\|_{L^{4}(\Omega)}\right\| \nabla h_{\mathrm{d}}\left(\varphi_{k}^{\varepsilon}\right)\right) \|_{L^{4}(\Omega)}\right) \\
& \lesssim\left\|\nabla \Delta \varphi_{k}^{\varepsilon}\right\|_{L^{2}(\Omega)}\left(\left\|\Delta \nabla \varphi_{k}^{\varepsilon}\right\|_{L^{2}(\Omega)}+\left\|\nabla \varphi_{k}^{\varepsilon}\right\|_{L^{2}(\Omega)}\right)\left\|\varphi_{k}^{\varepsilon}\right\|_{H^{2}(\Omega)} \\
& \quad+\left\|\nabla \Delta \varphi_{k}^{\varepsilon}\right\|_{L^{2}(\Omega)}\left\|\varphi_{k}^{\varepsilon}\right\|_{H^{2}(\Omega)}^{2},
\end{aligned}
$$

using $\left\|\nabla h_{\mathrm{d}}\left(\varphi_{k}^{\varepsilon}\right)\right\|_{L^{4}(\Omega)} \lesssim\left\|h_{\mathrm{d}}\left(\varphi_{k}^{\varepsilon}\right)\right\|_{H^{2}(\Omega)} \lesssim\left\|\varphi_{k}^{\varepsilon}\right\|_{H^{2}(\Omega)}$. Hence, there exists an absolute constant $C>0$, and for all $\eta>0$, there exists $C_{\eta}>0$ such that

$$
\begin{aligned}
&\left(\Delta^{2} \varphi_{k}^{\varepsilon} \mid \varphi_{k}^{\varepsilon} \wedge\left(\Delta \varphi_{k}^{\varepsilon}+h_{\mathrm{d}}\left(\varphi_{k}^{\varepsilon}\right)\right)\right)_{L^{2}(\Omega)} \leqslant \\
&\left(\eta+C\left\|\varphi_{k}^{\varepsilon}\right\|_{H^{2}(\Omega)}\right)\left\|\nabla \Delta \varphi_{k}^{\varepsilon}\right\|_{L^{2}(\Omega)}^{2}+C_{\eta}\left\|\varphi_{k}^{\varepsilon}\right\|_{H^{2}(\Omega)}^{4} .
\end{aligned}
$$

Summing up (4.11) and (4.12), one gets $C>0$ and, for all $\eta>0$, a constant $C_{\eta}>0$ (depending on $\left.\left\|n_{0}\right\|_{L^{\infty}\left((0, \infty), H^{2}(\Omega)\right)}\right)$ such that

$$
\begin{aligned}
I I_{1,1} \leqslant(\eta+ & \left.C\left\|\varphi_{k}^{\varepsilon}\right\|_{H^{2}(\Omega)}\right)\left\|\nabla \Delta \varphi_{k}^{\varepsilon}\right\|_{L^{2}(\Omega)}^{2} \\
& +C_{\eta}\left(1+\left\|\nabla \Delta n_{k}^{\varepsilon}\right\|_{L^{2}(\Omega)}^{2}+\left\|\varphi_{k}^{\varepsilon}\right\|_{H^{2}(\Omega)}^{2}\right)\left\|\varphi_{k}^{\varepsilon}\right\|_{H^{2}(\Omega)}^{2} .
\end{aligned}
$$

The second term is

$$
\begin{aligned}
I I_{1,2}= & -\left(\nabla \Delta \varphi_{k}^{\varepsilon} \mid \nabla \varphi_{k}^{\varepsilon} \wedge\left(\Delta n_{k}^{\varepsilon}+h_{\mathrm{d}}\left(n_{k}^{\varepsilon}\right)+h_{\mathrm{ext}}(t)\right)\right)_{L^{2}(\Omega)} \\
& -\left(\nabla \Delta \varphi_{k}^{\varepsilon} \mid \varphi_{k}^{\varepsilon} \wedge\left(\nabla \Delta n_{k}^{\varepsilon}+\nabla h_{\mathrm{d}}\left(\varphi_{k}^{\varepsilon}\right)+\nabla h_{\mathrm{ext}}(t)\right)\right)_{L^{2}(\Omega)} .
\end{aligned}
$$

Using Sobolev's inequalities again, we have, for all $\eta>0$, a constant $C_{\eta}$ (depending on $\left\|n_{0}\right\|_{L^{\infty}\left((0, \infty), H^{2}(\Omega)\right)}$ and $\left.\left\|h_{\text {ext }}\right\|_{L_{t, x}^{\infty}}\right)$ such that

$$
\begin{aligned}
&-\left(\nabla \Delta \varphi_{k}^{\varepsilon} \mid \nabla \varphi_{k}^{\varepsilon} \wedge\left(\Delta n_{k}^{\varepsilon}+h_{\mathrm{d}}\left(n_{k}^{\varepsilon}\right)+h_{\mathrm{ext}}(t)\right)\right)_{L^{2}(\Omega)} \leqslant\left\|\nabla \Delta \varphi_{k}^{\varepsilon}\right\|_{L^{2}(\Omega)} \times \\
& \times\left(\left\|\nabla \varphi_{k}^{\varepsilon}\right\|_{L^{4}(\Omega)}\left\|\Delta n_{k}^{\varepsilon}+h_{\mathrm{d}}\left(n_{k}^{\varepsilon}\right)\right\|_{L^{4}(\Omega)}+\left\|\nabla \varphi_{k}^{\varepsilon}\right\|_{L^{2}(\Omega)}\left\|h_{\mathrm{ext}}(t)\right\|_{L^{\infty}(\Omega)}\right) \\
& \leqslant C\left\|\nabla \Delta \varphi_{k}^{\varepsilon}\right\|_{L^{2}(\Omega)}\left\|\varphi_{k}^{\varepsilon}\right\|_{H^{2}(\Omega)} \\
&\left(\left\|\nabla \Delta n_{k}^{\varepsilon}\right\|_{L^{2}(\Omega)}+\left\|n_{k}^{\varepsilon}\right\|_{H^{2}(\Omega)}+\left\|h_{\mathrm{ext}}(t)\right\|_{L^{\infty}(\Omega)}\right) \\
& \quad \leqslant \eta\left\|\nabla \Delta \varphi_{k}^{\varepsilon}\right\|_{L^{2}(\Omega)}^{2}+C_{\eta}\left(1+\left\|\nabla \Delta n_{k}^{\varepsilon}\right\|_{L^{2}(\Omega)}^{2}\right)\left\|\varphi_{k}^{\varepsilon}\right\|_{H^{2}(\Omega)}^{2} .
\end{aligned}
$$


In the same way, for all $\eta>0$, there is $C_{\eta}>0$ (depending on $\left\|n_{0}\right\|_{L^{\infty}\left((0, \infty), H^{2}(\Omega)\right)}$ and $\left.\left\|\nabla h_{\text {ext }}\right\|_{L_{t, x}^{\infty}}\right)$ such that

$$
\begin{aligned}
& -\left(\nabla \Delta \varphi_{k}^{\varepsilon} \mid \varphi_{k}^{\varepsilon} \wedge\left(\nabla \Delta n_{k}^{\varepsilon}+\nabla h_{\mathrm{d}}\left(\varphi_{k}^{\varepsilon}\right)+\nabla h_{\mathrm{ext}}(t)\right)\right)_{L^{2}(\Omega)} \leqslant \\
& \leqslant \eta\left\|\nabla \Delta \varphi_{k}^{\varepsilon}\right\|_{L^{2}(\Omega)}^{2}+C_{\eta}\left(1+\left\|\nabla \Delta n_{k}^{\varepsilon}\right\|_{L^{2}(\Omega)}^{2}\right)\left\|\varphi_{k}^{\varepsilon}\right\|_{H^{2}(\Omega)}^{2} .
\end{aligned}
$$

Summing up (4.14) and (4.15), we get, for all $\eta>0$, a constant $C_{\eta}>0$ (depending on $\left\|h_{\text {ext }}\right\|_{L_{t, x}^{\infty}},\left\|\nabla h_{\text {ext }}\right\|_{L_{t, x}^{\infty}}$ and $\left.\left\|n_{0}\right\|_{L^{\infty}\left((0, \infty), H^{2}(\Omega)\right)}\right)$ such that

$$
I I_{1,2} \leqslant \eta\left\|\nabla \Delta \varphi_{k}^{\varepsilon}\right\|_{L^{2}(\Omega)}^{2}+C_{\eta}\left(1+\left\|\nabla \Delta n_{k}^{\varepsilon}\right\|_{L^{2}(\Omega)}^{2}\right)\left\|\varphi_{k}^{\varepsilon}\right\|_{H^{2}(\Omega)}^{2} .
$$

The third term is

$$
I I_{1,3}=\left(\Delta^{2} \varphi_{k}^{\varepsilon} \mid n_{k}^{\varepsilon} \wedge\left(h_{\mathrm{ext}}(t)-h_{\mathrm{ext}}(0)\right)\right)_{L^{2}(\Omega)} .
$$

Integrating two times by parts, it is easily estimated, thanks to a constant $C$ depending on $\left\|\partial_{t} h_{\text {ext }}\right\|_{L_{t}^{\infty} W_{x}^{2, \infty}}$, as

$$
I I_{1,3} \leqslant C t\left\|n_{k}^{\varepsilon}\right\|_{H^{2}(\Omega)}\left\|\varphi_{k}^{\varepsilon}\right\|_{H^{2}(\Omega)} .
$$

This gives finally, summing up (4.13), (4.16) and (4.17): there is $C>0$, and for all $\eta>0$, there is $C_{\eta}>0$ (depending on $\eta,\left\|n_{0}\right\|_{L^{\infty}\left((0, \infty), H^{2}(\Omega)\right.}$ ), $\left\|h_{\text {ext }}\right\|_{L_{t}^{\infty} W_{x}^{1, \infty}}$ and $\left\|\partial_{t} h_{\text {ext }}\right\|_{\left.L_{t}^{\infty} W_{x}^{2, \infty}\right)}$, such that

$$
\begin{aligned}
I I_{1} \leqslant & \left(\eta+C\left\|\varphi_{k}^{\varepsilon}\right\|_{H^{2}(\Omega)}\right)\left\|\nabla \Delta \varphi_{k}^{\varepsilon}\right\|_{L^{2}(\Omega)}^{2} \\
& +C_{\eta}\left(\left(1+\left\|\nabla \Delta n_{k}^{\varepsilon}\right\|_{L^{2}(\Omega)}^{2}+\left\|\varphi_{k}^{\varepsilon}\right\|_{H^{2}(\Omega)}^{2}\right)\left\|\varphi_{k}^{\varepsilon}\right\|_{H^{2}(\Omega)}^{2}+t\left\|\varphi_{k}^{\varepsilon}\right\|_{H^{2}(\Omega)}\right) .
\end{aligned}
$$

Estimating $I I_{2}$. Split

$$
I I_{2}=I I_{2,1}+I I_{2,2}
$$

with

$$
\begin{aligned}
I I_{2,1} & =\left(\Delta^{2} \varphi_{k}^{\varepsilon} \mid\left(\left|\nabla m_{k}^{\varepsilon}\right|^{2}-\left|\nabla n_{k}^{\varepsilon}\right|^{2}\right)\left(n_{k}^{\varepsilon}+\varphi_{k}^{\varepsilon}\right)\right)_{L^{2}(\Omega)} \\
& =\left(\Delta^{2} \varphi_{k}^{\varepsilon} \mid \nabla \varphi_{k}^{\varepsilon} \cdot\left(\nabla \varphi_{k}^{\varepsilon}+2 \nabla n_{k}^{\varepsilon}\right) m_{k}^{\varepsilon}\right)_{L^{2}(\Omega)} \\
I I_{2,2} & =\left(\left.\Delta^{2} \varphi_{k}^{\varepsilon}|| \nabla n_{k}^{\varepsilon}\right|^{2}\left(m_{k}^{\varepsilon}-n_{k}^{\varepsilon}\right)\right)_{L^{2}(\Omega)}=\left(\left.\Delta^{2} \varphi_{k}^{\varepsilon}|| \nabla n_{k}^{\varepsilon}\right|^{2} \varphi_{k}^{\varepsilon}\right)_{L^{2}(\Omega)} .
\end{aligned}
$$

Then, using in particular the Sobolev inequality from Lemma 3.2

$$
\left\|\nabla n_{k}^{\varepsilon}\right\|_{L^{\infty}(\Omega)} \lesssim\left\|\nabla n_{k}^{\varepsilon}\right\|_{L^{2}(\Omega)}+\left\|\nabla \Delta n_{k}^{\varepsilon}\right\|_{L^{2}(\Omega)},
$$


we get:

$$
\begin{aligned}
& I I_{2,1}=-\left(\nabla \Delta \varphi_{k}^{\varepsilon} \mid \Delta \varphi_{k}^{\varepsilon} \cdot\left(\nabla \varphi_{k}^{\varepsilon}+2 \nabla n_{k}^{\varepsilon}\right)\left(n_{k}^{\varepsilon}+\varphi_{k}^{\varepsilon}\right)\right)_{L^{2}(\Omega)} \\
& -\left(\nabla \Delta \varphi_{k}^{\varepsilon} \mid \nabla \varphi_{k}^{\varepsilon} \cdot\left(\Delta \varphi_{k}^{\varepsilon}+2 \Delta n_{k}^{\varepsilon}\right)\left(n_{k}^{\varepsilon}+\varphi_{k}^{\varepsilon}\right)\right)_{L^{2}(\Omega)} \\
& -\left(\nabla \Delta \varphi_{k}^{\varepsilon} \mid \nabla \varphi_{k}^{\varepsilon} \cdot\left(\nabla \varphi_{k}^{\varepsilon}+2 \nabla n_{k}^{\varepsilon}\right)\left(\nabla n_{k}^{\varepsilon}+\nabla \varphi_{k}^{\varepsilon}\right)\right)_{L^{2}(\Omega)} \\
& \leqslant\left\|\nabla \Delta \varphi_{k}^{\varepsilon}\right\|_{L^{2}(\Omega)}\left\|\Delta \varphi_{k}^{\varepsilon}\right\|_{L^{2}(\Omega)} \times \\
& \times\left(\left\|\nabla \varphi_{k}^{\varepsilon}\right\|_{L^{\infty}(\Omega)}+2\left\|\nabla n_{k}^{\varepsilon}\right\|_{L^{\infty}(\Omega)}\right)\left(\left\|n_{k}^{\varepsilon}\right\|_{L^{\infty}(\Omega)}+\left\|\varphi_{k}^{\varepsilon}\right\|_{L^{\infty}(\Omega)}\right) \\
& +\left\|\nabla \Delta \varphi_{k}^{\varepsilon}\right\|_{L^{2}(\Omega)}\left\|\nabla \varphi_{k}^{\varepsilon}\right\|_{L^{4}(\Omega)} \times \\
& \times\left(\left\|\Delta \varphi_{k}^{\varepsilon}\right\|_{L^{4}(\Omega)}+2\left\|\Delta n_{k}^{\varepsilon}\right\|_{L^{4}(\Omega)}\right)\left(\left\|n_{k}^{\varepsilon}\right\|_{L^{\infty}(\Omega)}+\left\|\varphi_{k}^{\varepsilon}\right\|_{L^{\infty}(\Omega)}\right) \\
& +\left\|\nabla \Delta \varphi_{k}^{\varepsilon}\right\|_{L^{2}(\Omega)}\left\|\nabla \varphi_{k}^{\varepsilon}\right\|_{L^{6}(\Omega)} \times \\
& \times\left(\left\|\nabla \varphi_{k}^{\varepsilon}\right\|_{L^{6}(\Omega)}+2\left\|\nabla n_{k}^{\varepsilon}\right\|_{L^{6}(\Omega)}\right)\left(\left\|\nabla n_{k}^{\varepsilon}\right\|_{L^{6}(\Omega)}+\left\|\nabla \varphi_{k}^{\varepsilon}\right\|_{L^{6}(\Omega)}\right) \\
& \lesssim\left\|\nabla \Delta \varphi_{k}^{\varepsilon}\right\|_{L^{2}(\Omega)}\left\|\varphi_{k}^{\varepsilon}\right\|_{H^{2}(\Omega)}\left(\left\|n_{k}^{\varepsilon}\right\|_{H^{2}(\Omega)}+\left\|\varphi_{k}^{\varepsilon}\right\|_{H^{2}(\Omega)}\right) \times \\
& \times\left(\left\|\nabla \varphi_{k}^{\varepsilon}\right\|_{L^{2}(\Omega)}+\left\|\nabla \Delta \varphi_{k}^{\varepsilon}\right\|_{L^{2}(\Omega)}+\left\|\nabla n_{k}^{\varepsilon}\right\|_{L^{2}(\Omega)}+\left\|\nabla \Delta n_{k}^{\varepsilon}\right\|_{L^{2}(\Omega)}\right) \\
& +\left\|\nabla \Delta \varphi_{k}^{\varepsilon}\right\|_{L^{2}(\Omega)}\left\|\varphi_{k}^{\varepsilon}\right\|_{H^{2}(\Omega)}\left(\left\|n_{k}^{\varepsilon}\right\|_{H^{2}(\Omega)}+\left\|\varphi_{k}^{\varepsilon}\right\|_{H^{2}(\Omega)}\right) \times \\
& \times\left(\left\|\Delta \varphi_{k}^{\varepsilon}\right\|_{L^{2}(\Omega)}+\left\|\nabla \Delta \varphi_{k}^{\varepsilon}\right\|_{L^{2}(\Omega)}+\left\|\Delta n_{k}^{\varepsilon}\right\|_{L^{2}(\Omega)}+\left\|\nabla \Delta n_{k}^{\varepsilon}\right\|_{L^{2}(\Omega)}\right) \\
& +\left\|\nabla \Delta \varphi_{k}^{\varepsilon}\right\|_{L^{2}(\Omega)}\left\|\varphi_{k}^{\varepsilon}\right\|_{H^{2}(\Omega)}\left(\left\|n_{k}^{\varepsilon}\right\|_{H^{2}(\Omega)}+\left\|\varphi_{k}^{\varepsilon}\right\|_{H^{2}(\Omega)}\right)^{2} \\
& \lesssim\left\|\nabla \Delta \varphi_{k}^{\varepsilon}\right\|_{L^{2}(\Omega)}\left\|\varphi_{k}^{\varepsilon}\right\|_{H^{2}(\Omega)}\left(\left\|n_{k}^{\varepsilon}\right\|_{H^{2}(\Omega)}+\left\|\varphi_{k}^{\varepsilon}\right\|_{H^{2}(\Omega)}\right) \times \\
& \times\left(\left\|\varphi_{k}^{\varepsilon}\right\|_{H^{2}(\Omega)}+\left\|\nabla \Delta \varphi_{k}^{\varepsilon}\right\|_{L^{2}(\Omega)}+\left\|n_{k}^{\varepsilon}\right\|_{H^{2}(\Omega)}+\left\|\nabla \Delta n_{k}^{\varepsilon}\right\|_{L^{2}(\Omega)}\right) \\
& \lesssim\left(\eta+\left\|\varphi_{k}^{\varepsilon}\right\|_{H^{2}(\Omega)}\left(\left\|n_{k}^{\varepsilon}\right\|_{H^{2}(\Omega)}+\left\|\varphi_{k}^{\varepsilon}\right\|_{H^{2}(\Omega)}\right)\right)\left\|\nabla \Delta \varphi_{k}^{\varepsilon}\right\|_{L^{2}(\Omega)}^{2} \\
& +C_{\eta}\left\|\varphi_{k}^{\varepsilon}\right\|_{H^{2}(\Omega)}^{2}\left(\left\|\varphi_{k}^{\varepsilon}\right\|_{H^{2}(\Omega)}+\left\|n_{k}^{\varepsilon}\right\|_{H^{3}(\Omega)}+\left\|\nabla \Delta n_{k}^{\varepsilon}\right\|_{L^{2}(\Omega)}\right)^{2} \times \\
& \times\left(\left\|n_{k}^{\varepsilon}\right\|_{H^{2}(\Omega)}+\left\|\varphi_{k}^{\varepsilon}\right\|_{H^{2}(\Omega)}\right)^{2},
\end{aligned}
$$

for all $\eta>0$, for some $C_{\eta}>0$. 
Also, for all $\eta>0$, there is $C_{\eta}>0$ such that

$$
\begin{aligned}
& I I_{2,2}=-\left(\nabla \Delta \varphi_{k}^{\varepsilon} \mid 2 \nabla n_{k}^{\varepsilon} \Delta n_{k}^{\varepsilon} \varphi_{k}^{\varepsilon}\right)_{L^{2}(\Omega)}-\left(\left.\nabla \Delta \varphi_{k}^{\varepsilon}|| \nabla n_{k}^{\varepsilon}\right|^{2} \nabla \varphi_{k}^{\varepsilon}\right)_{L^{2}(\Omega)} \\
& \leqslant\left\|\nabla \Delta \varphi_{k}^{\varepsilon}\right\|_{L^{2}(\Omega)}\left(2\left\|\nabla n_{k}^{\varepsilon}\right\|_{L^{\infty}(\Omega)}\left\|\Delta n_{k}^{\varepsilon}\right\|_{L^{2}(\Omega)}\left\|\varphi_{k}^{\varepsilon}\right\|_{L^{\infty}(\Omega)}\right. \\
& \left.+\left\|\nabla n_{k}^{\varepsilon}\right\|_{L^{6}(\Omega)}^{2}\left\|\nabla \varphi_{k}^{\varepsilon}\right\|_{L^{6}(\Omega)}\right) \\
& \lesssim \eta\left\|\nabla \Delta \varphi_{k}^{\varepsilon}\right\|_{L^{2}(\Omega)}^{2} \\
& +C_{\eta}\left(\left\|n_{k}^{\varepsilon}\right\|_{H^{2}(\Omega)}^{2}+\left\|\nabla \Delta n_{k}^{\varepsilon}\right\|_{L^{2}(\Omega)}^{2}+1\right)\left\|n_{k}^{\varepsilon}\right\|_{H^{2}(\Omega)}^{2}\left\|\varphi_{k}^{\varepsilon}\right\|_{H^{2}(\Omega)}^{2} .
\end{aligned}
$$

Summing up (4.19) and (4.20), we get: there is $C>0$, and for all $\eta>0$, there is $C_{\eta}>0$ (with $C$ and $C_{\eta}$ depending on $\left.\left\|n_{0}\right\|_{L^{\infty}\left((0, \infty), H^{2}(\Omega)\right)}\right)$ such that

$$
\begin{aligned}
I I_{2} \leqslant C & \left(\eta+\left\|\varphi_{k}^{\varepsilon}\right\|_{H^{2}(\Omega)}\left(1+\left\|\varphi_{k}^{\varepsilon}\right\|_{H^{2}(\Omega)}\right)\right)\left\|\nabla \Delta \varphi_{k}^{\varepsilon}\right\|_{L^{2}(\Omega)}^{2} \\
& +C_{\eta}\left\|\varphi_{k}^{\varepsilon}\right\|_{H^{2}(\Omega)}^{2}\left(1+\left\|\varphi_{k}^{\varepsilon}\right\|_{H^{2}(\Omega)}^{2}\right)\left(1+\left\|\varphi_{k}^{\varepsilon}\right\|_{H^{2}(\Omega)}^{2}+\left\|\nabla \Delta n_{k}^{\varepsilon}\right\|_{L^{2}(\Omega)}^{2}\right) .
\end{aligned}
$$

Estimating $I_{3}$. Now,

$$
I I_{3}=I I_{3,1}+I I_{3,2}
$$

with

$$
\begin{aligned}
& I I_{3,1}=-\alpha\left(\Delta^{2} \varphi_{k}^{\varepsilon} \mid m_{k}^{\varepsilon} \wedge\left(m_{k}^{\varepsilon} \wedge h_{\mathrm{d}}\left(m_{k}^{\varepsilon}\right)\right)-n_{k}^{\varepsilon} \wedge\left(n_{k}^{\varepsilon} \wedge h_{\mathrm{d}}\left(n_{k}^{\varepsilon}\right)\right)\right)_{L^{2}(\Omega)}, \\
& I I_{3,2}=-\alpha\left(\Delta^{2} \varphi_{k}^{\varepsilon} \mid m_{k}^{\varepsilon} \wedge\left(m_{k}^{\varepsilon} \wedge h_{\mathrm{ext}}(t)\right)-n_{k}^{\varepsilon} \wedge\left(n_{k}^{\varepsilon} \wedge h_{\mathrm{ext}}(0)\right)\right)_{L^{2}(\Omega)} .
\end{aligned}
$$

Concerning $I I_{3,1}$, first write $m_{k}^{\varepsilon}=n_{k}^{\varepsilon}+\varphi_{k}^{\varepsilon}$, then integrate once by parts, so that $I I_{3,1}$ takes the form of a $L^{2}$ scalar product between $\nabla \Delta \varphi_{k}^{\varepsilon}$ and a sum of terms $\nabla(a b c)$, where $a, b, c$ may be $n_{k}^{\varepsilon}$ (or $\left.h_{\mathrm{d}}\left(n_{k}^{\varepsilon}\right)\right)$ or $\varphi_{k}^{\varepsilon}$ (or $h_{\mathrm{d}}\left(\varphi_{k}^{\varepsilon}\right)$ ), and at least one of them is $n_{k}^{\varepsilon}$ (or $h_{\mathrm{d}}\left(n_{k}^{\varepsilon}\right)$ ). Estimating each of $a, b, c$ and their gradients in $L^{6}$, one gets: for all $\eta>0$, there is $C_{\eta}>0$ such that

$$
I I_{3,1} \leqslant \eta\left\|\nabla \Delta \varphi_{k}^{\varepsilon}\right\|_{L^{2}(\Omega)}^{2}+C_{\eta}\left(1+\left\|n_{k}^{\varepsilon}\right\|_{H^{2}(\Omega)}^{2}+\left\|\varphi_{k}^{\varepsilon}\right\|_{H^{2}(\Omega)}^{2}\right)^{2}\left\|\varphi_{k}^{\varepsilon}\right\|_{H^{2}(\Omega)}^{2} .
$$

Then, split $I I_{3,2}$,

$$
\begin{aligned}
I I_{3,2}= & -\alpha\left(\Delta^{2} \varphi_{k}^{\varepsilon} \mid m_{k}^{\varepsilon} \wedge\left(m_{k}^{\varepsilon} \wedge\left(h_{\mathrm{ext}}(t)-h_{\mathrm{ext}}(0)\right)\right)\right)_{L^{2}(\Omega)} \\
& -\alpha\left(\Delta^{2} \varphi_{k}^{\varepsilon} \mid m_{k}^{\varepsilon} \wedge\left(m_{k}^{\varepsilon} \wedge h_{\mathrm{ext}}(0)\right)-n_{k}^{\varepsilon} \wedge\left(n_{k}^{\varepsilon} \wedge h_{\mathrm{ext}}(0)\right)\right)_{L^{2}(\Omega)} .
\end{aligned}
$$

The second term is estimated as $I I_{3,1}$. The first one is split into a sum involving $n_{k}^{\varepsilon} \wedge\left(n_{k}^{\varepsilon} \wedge\left(h_{\text {ext }}(t)-h_{\text {ext }}(0)\right)\right)$, and products of $h_{\text {ext }}(t)$ with two terms, 
one of them being $\varphi_{k}^{\varepsilon}$, and the other, $\varphi_{k}^{\varepsilon}$ or $n_{k}^{\varepsilon}$. This leads to: for all $\eta>0$, there is $C_{\eta}>0$ (also depending on $h_{\text {ext }}$ ) such that

$$
\begin{gathered}
I I_{3,2} \leqslant \eta\left\|\nabla \Delta \varphi_{k}^{\varepsilon}\right\|_{L^{2}(\Omega)}^{2}+C_{\eta}\left(\left(1+\left\|n_{k}^{\varepsilon}\right\|_{H^{2}(\Omega)}^{2}+\left\|\varphi_{k}^{\varepsilon}\right\|_{H^{2}(\Omega)}^{2}\right)\left\|\varphi_{k}^{\varepsilon}\right\|_{H^{2}(\Omega)}^{2}\right. \\
\left.+t\left\|n_{k}^{\varepsilon}\right\|_{H^{2}(\Omega)}^{2}\left\|\varphi_{k}^{\varepsilon}\right\|_{H^{2}(\Omega)}\right) .
\end{gathered}
$$

Finally, summing up (4.22) and (4.23), we have: for all $\eta>0$, there is $C_{\eta}>0$ (depending on $\left.\left\|n_{0}\right\|_{L^{\infty}\left((0, \infty), H^{2}(\Omega)\right)}\right)$ such that

$$
\begin{aligned}
I I_{3} \leqslant & \eta\left\|\nabla \Delta \varphi_{k}^{\varepsilon}\right\|_{L^{2}(\Omega)}^{2} \\
& +C_{\eta}\left(\left(1+\left\|\varphi_{k}^{\varepsilon}\right\|_{H^{2}(\Omega)}^{2}\right)^{2}\left\|\varphi_{k}^{\varepsilon}\right\|_{H^{2}(\Omega)}^{2}+t\left\|\varphi_{k}^{\varepsilon}\right\|_{H^{2}(\Omega)}\right) .
\end{aligned}
$$

Estimating $I I_{4}$. Integrating once by parts, we get

$$
I I_{4}=-\left(\nabla \Delta \varphi_{k}^{\varepsilon} \mid \nabla\left[P_{k}, \mathcal{F}(0, \cdot)\right]\left(n^{\varepsilon}\right)\right)_{L^{2}(\Omega)} .
$$

Thus, for all $\eta>0$, there exists $C_{\eta}>0$ such that

$$
I I_{4} \leqslant \eta\left\|\nabla \Delta \varphi_{k}^{\varepsilon}\right\|_{L^{2}(\Omega)}^{2}+C_{\eta} r_{k, 1}^{\varepsilon}
$$

with

$$
r_{k, 1}^{\varepsilon}=\left\|\nabla\left[P_{k}, \mathcal{F}(0, \cdot)\right]\left(n^{\varepsilon}\right)\right\|_{L^{2}(\Omega)}^{2} \underset{k \rightarrow \infty}{\longrightarrow} 0 \text { in } L^{\infty}(0, T) \text { for all } T>0 \text {, with } \varepsilon \text { fixed, }
$$

thanks to Lemma 4.1.

\subsubsection{Conclusion}

¿From (4.18), (4.21), (4.24) and (4.25), we deduce that there is a constant $C>0$ (depending on $\left.\left\|n_{0}\right\|_{L^{\infty}\left((0, \infty), H^{3}(\Omega)\right)}\right)$, and for all $\eta>0$, there is $C_{\eta}>$ 0 (depending on $\eta,\left\|n_{0}\right\|_{L^{\infty}\left((0, \infty), H^{3}(\Omega)\right)},\left\|h_{\text {ext }}\right\|_{L_{t}^{\infty} W_{x}^{1, \infty}}$ and $\left\|\partial_{t} h_{\text {ext }}\right\|_{L_{t}^{\infty} W_{x}^{2, \infty}}$ ), such that

$$
\begin{aligned}
& \frac{\varepsilon}{2} \frac{\mathrm{d}}{\mathrm{dt}}\left(\left\|\Delta \varphi_{k}^{\varepsilon}\right\|_{L^{2}(\Omega)}^{2}\right) \\
& +\left(\alpha-C\left(\eta+\left\|\varphi_{k}^{\varepsilon}\right\|_{H^{2}(\Omega)}\left(1+\left\|\varphi_{k}^{\varepsilon}\right\|_{H^{2}(\Omega)}\right)\right)\right)\left\|\nabla \Delta \varphi_{k}^{\varepsilon}\right\|_{L^{2}(\Omega)}^{2} \\
& \quad \leqslant C_{\eta}\left(\left\|\varphi_{k}^{\varepsilon}\right\|_{H^{2}(\Omega)}^{2}\left(1+\left\|\varphi_{k}^{\varepsilon}\right\|_{H^{2}(\Omega)}^{2}\right)\left(1+\left\|\varphi_{k}^{\varepsilon}\right\|_{H^{2}(\Omega)}^{2}+\left\|\nabla \Delta n_{k}^{\varepsilon}\right\|_{L^{2}(\Omega)}^{2}\right)\right. \\
& \left.+t\left\|\varphi_{k}^{\varepsilon}\right\|_{H^{2}(\Omega)}+r_{k, 1}^{\varepsilon}\right)
\end{aligned}
$$

Sum up (4.10) and (4.26), to get: there is a constant $C>0$ (depending on $\left.\left\|n_{0}\right\|_{L^{\infty}\left((0, \infty), H^{3}(\Omega)\right)}\right)$, and for all $\eta>0$, there is $C_{\eta}>0$ (depending on $\eta$, 
$\left\|n_{0}\right\|_{L^{\infty}\left((0, \infty), H^{2}(\Omega)\right)},\left\|h_{\text {ext }}\right\|_{L_{t}^{\infty} W_{x}^{1, \infty}}$ and $\left\|\partial_{t} h_{\text {ext }}\right\|_{\left.L_{t}^{\infty} W_{x}^{2, \infty}\right)}$, such that

$$
\begin{gathered}
\frac{\varepsilon}{2} \frac{\mathrm{d}}{\mathrm{dt}}\left(\left\|\varphi_{k}^{\varepsilon}\right\|_{H^{2}(\Omega)}^{2}\right)+\left(\alpha-C\left(\eta+\left\|\varphi_{k}^{\varepsilon}\right\|_{H^{2}(\Omega)}\left(1+\left\|\varphi_{k}^{\varepsilon}\right\|_{H^{2}(\Omega)}\right)\right)\right)\left\|\nabla \Delta \varphi_{k}^{\varepsilon}\right\|_{H^{2}(\Omega)}^{2} \\
\leqslant C_{\eta}\left(\left\|\varphi_{k}^{\varepsilon}\right\|_{H^{2}(\Omega)}^{2}\left(1+\left\|\varphi_{k}^{\varepsilon}\right\|_{H^{2}(\Omega)}^{2}\right)\left(1+\left\|\varphi_{k}^{\varepsilon}\right\|_{H^{2}(\Omega)}^{2}+\left\|\nabla \Delta n_{k}^{\varepsilon}\right\|_{L^{2}(\Omega)}^{2}\right)\right. \\
\left.+t\left\|\varphi_{k}^{\varepsilon}\right\|_{H^{2}(\Omega)}+\tilde{r}_{k}^{\varepsilon}\right)
\end{gathered}
$$

with $\tilde{r}_{k}^{\varepsilon}=\left\|\left[P_{k}, \mathcal{F}(0, \cdot)\right]\left(n^{\varepsilon}\right)\right\|_{H^{1}(\Omega)}^{2} \underset{k \rightarrow \infty}{\longrightarrow} 0$ in $L^{\infty}(0, T)$ for all $T>0$, with $\varepsilon$ fixed.

Now, apply the following Gronwall lemma (the proof of which is postponed to Section 6.3).

Lemma 4.2. There is a constant $K>0$ (depending on $n_{0}$ and $h_{\mathrm{ext}}$ ) such that, for all $c \in(0,1 / K)$, setting $t_{\varepsilon}=c \varepsilon \ln (1 / \varepsilon)$, there is $\varepsilon_{0}=\varepsilon_{0}(\alpha, c, K)$ such that (4.27) implies:

$$
\begin{aligned}
\forall \varepsilon & \in\left(0, \varepsilon_{0}\right], \quad \exists \underline{k}(\varepsilon) \in \mathbb{N}^{\star}, \quad \forall k \geqslant \underline{k}(\varepsilon), \\
& \sup _{\left[0, t_{\varepsilon}\right]}\left\|\varphi_{k}^{\varepsilon}\right\|_{H^{2}(\Omega)}^{2} \leqslant\left(\frac{\varepsilon^{1-c K}}{K}+K\left\|\tilde{r}_{k}^{\varepsilon}\right\|_{L^{1}\left(0, t_{\varepsilon_{0}}\right)} \varepsilon^{-1-c K}\right) e^{K\left\|\nabla \Delta P_{k} n_{0}\right\|_{L^{2}((0, \infty) \times \Omega)}^{2} .}
\end{aligned}
$$

\subsubsection{Passing to the limit $k \rightarrow \infty$}

For each $\varepsilon \in\left(0, \varepsilon_{0}\right]$ fixed, by Lemma 4.2 , the sequence $\left(\varphi_{k}^{\varepsilon}\right)_{k \in \mathbb{N}^{\star}}$ is bounded in $L^{\infty}\left(\left(0, t_{\varepsilon}\right), H^{2}(\Omega)\right)$. Equation (4.5) then implies that the sequence $\left(\partial_{t} \varphi_{k}^{\varepsilon}\right)_{k \in \mathbb{N}^{*}}$ is bounded in $L^{\infty}\left(\left(0, t_{\varepsilon}\right), L^{2}(\Omega)\right)$. Furthermore, (4.27) shows that $\left(\varphi_{k}^{\varepsilon}\right)_{k \in \mathbb{N}^{*}}$ is also bounded in $L^{2}\left(\left(0, t_{\varepsilon}\right), H^{3}(\Omega)\right)$. Aubin's Lemma (see [2], [10]) then implies that there is a subsequence of $\left(\varphi_{k}^{\varepsilon}\right)_{k \in \mathbb{N}^{\star}}$ converging in $L^{2}\left(\left(0, t_{\varepsilon}\right), H^{2}(\Omega)\right)$ towards some $\varphi^{\varepsilon}$.

Up to a subsequence, we may assume that $\left(\partial_{t} \varphi_{k}^{\varepsilon}\right)_{k \in \mathbb{N}^{\star}}$ also converges weakly in $L^{2}\left(\left(0, t_{\varepsilon}\right), L^{2}(\Omega)\right)$ towards $\partial_{t} \varphi^{\varepsilon}$. As $k$ goes to $\infty, P_{k} n^{\varepsilon}$ converges towards $n^{\varepsilon}$ in $C\left(\left[0, t_{\varepsilon}\right], H^{2}(\Omega)\right) \cap H^{1}\left(\left(0, t_{\varepsilon}\right), L^{2}(\Omega)\right)$. Thus, $\left(m_{k}^{\varepsilon}\right)_{k \in \mathbb{N}^{\star}}$ converges towards some $m^{\varepsilon}$ in $L^{2}\left(\left(0, t_{\varepsilon}\right), H^{2}(\Omega)\right)$, with $\left(\partial_{t} m_{k}^{\varepsilon}\right)_{k \in \mathbb{N}^{\star}}$ converging weakly in $L^{2}\left(\left(0, t_{\varepsilon}\right), L^{2}(\Omega)\right)$ towards $\partial_{t} m^{\varepsilon}$. This is enough to pass to the limit in (4.4), so that $m^{\varepsilon}$ is solution to (4.2). With $\varepsilon$ fixed, showing that $m^{\varepsilon}$ is continuous in time with values in $H^{2}$ is standard, as well as uniqueness and stability properties: see [4], or [1].

Finally, passing to the limit in Lemma 4.2 yields:

$$
\sup _{\left[0, t_{\varepsilon}\right]}\left\|\varphi^{\varepsilon}\right\|_{H^{2}(\Omega)}^{2} \leqslant \frac{\varepsilon^{1-c K}}{K} e^{K\left\|\nabla \Delta n_{0}\right\|_{L^{2}((0, \infty) \times \Omega)}^{2}}
$$

which we write

$$
\sup _{\left[0, t_{\varepsilon}\right]}\left\|\varphi^{\varepsilon}\right\|_{H^{2}(\Omega)}^{2} \leqslant K^{\prime} \varepsilon^{1-c K}
$$




\subsection{Second step: following the slow dynamics after $t_{\varepsilon}$}

¿From the local-in-time existence result, we know that, for each $\varepsilon \in\left(0, \varepsilon_{0}\right)$, there is $t^{\varepsilon}>t_{\varepsilon}$ such that $m^{\varepsilon}$ exists, as a solution to $(4.2)$, in $C\left(\left[0, t^{\varepsilon}\right], H^{2}(\Omega)\right) \cap$ $L^{2}\left(\left(0, t^{\varepsilon}\right), H^{3}(\Omega)\right)$. We shall show, via a priori estimates, that $t^{\varepsilon} \geqslant T$ (possibly reducing $\left.\varepsilon_{0}\right)$.

¿From (3.1), and with $\mathcal{F}$ from (4.3), we deduce that, on $[0, T] \times \Omega$,

$$
\varepsilon \partial_{t} m_{\mathrm{eq}}-\alpha \Delta m_{\mathrm{eq}}=\mathcal{F}\left(t, m_{\mathrm{eq}}\right)+\varepsilon \partial_{t} m_{\mathrm{eq}} .
$$

Substracting to $(4.2)$, we get $\left(\right.$ on $\left.\left[0, t^{\varepsilon}\right] \times \Omega\right)$ :

$$
\left\{\begin{array}{l}
\left(\varepsilon \partial_{t}-\alpha \Delta\right)\left(m^{\varepsilon}-m_{\mathrm{eq}}\right)=\left(\mathcal{L}\left(m_{\mathrm{eq}}\right)+\mathcal{R}\left(m_{\mathrm{eq}}\right)\right)\left(m^{\varepsilon}-m_{\mathrm{eq}}\right)+\varepsilon \partial_{t} m_{\mathrm{eq}}, \\
\partial_{\nu}\left(m^{\varepsilon}-m_{\mathrm{eq}}\right)_{\left.\right|_{\partial \Omega}}=0,
\end{array}\right.
$$

and we consider the associated Cauchy problem with data given at time $t_{\varepsilon}$. The data at time $t_{\varepsilon}=c \varepsilon \ln (1 / \varepsilon)$ satisfy (using (4.28) and (4.1)):

$$
\begin{aligned}
\left\|\left(m^{\varepsilon}-m_{\mathrm{eq}}\right)\left(t_{\varepsilon}\right)\right\|_{H^{2}(\Omega)} \leqslant \|\left(m^{\varepsilon}-n^{\varepsilon}\right) & \left(t_{\varepsilon}\right)\left\|_{H^{2}(\Omega)}+\right\| n^{\varepsilon}\left(t_{\varepsilon}\right)-m_{\mathrm{eq}}(0) \|_{H^{2}(\Omega)} \\
& +\left\|m_{\mathrm{eq}}(0)-m_{\mathrm{eq}}\left(t_{\varepsilon}\right)\right\|_{H^{2}(\Omega)} \\
\leqslant K^{\prime} \varepsilon^{1-c K}+ & \left\|n_{0}(c \ln (1 / \varepsilon))-m_{\mathrm{eq}}(0)\right\|_{H^{2}(\Omega)} \\
& +\left\|m_{\mathrm{eq}}(0)-m_{\mathrm{eq}}\left(t_{\varepsilon}\right)\right\|_{H^{2}(\Omega)} \underset{\varepsilon \rightarrow 0}{\longrightarrow} 0 .
\end{aligned}
$$

Here, for all $\delta \in H^{2}(\Omega)$ and $t \in[0, T]$,

$$
\begin{aligned}
\mathcal{L}\left(t, m_{\mathrm{eq}}(t)\right) \delta= & \alpha\left|\nabla m_{\mathrm{eq}}(t)\right|^{2} \delta+2 \alpha\left(\nabla m_{\mathrm{eq}}(t) \cdot \nabla \delta\right) m_{\mathrm{eq}}(t) \\
& +\delta \wedge h_{T}\left(t, m_{\mathrm{eq}}(t)\right)+m_{\mathrm{eq}}(t) \wedge\left(\Delta \delta+h_{\mathrm{d}}(\delta)\right) \\
& -\alpha \delta \wedge\left(m_{\mathrm{eq}}(t) \wedge\left(h_{\mathrm{d}}\left(m_{\mathrm{eq}}(t)\right)+h_{\mathrm{ext}}(t)\right)\right) \\
& -\alpha m_{\mathrm{eq}}(t) \wedge\left(\delta \wedge\left(h_{\mathrm{d}}\left(m_{\mathrm{eq}}(t)\right)+h_{\mathrm{ext}}(t)\right)\right) \\
& -\alpha m_{\mathrm{eq}}(t) \wedge\left(m_{\mathrm{eq}}(t) \wedge h_{\mathrm{d}}(\delta)\right),
\end{aligned}
$$

and

$$
\begin{aligned}
\mathcal{R}\left(t, m_{\mathrm{eq}}(t)\right) & (\delta)=2 \alpha\left(\nabla m_{\mathrm{eq}}(t) \cdot \nabla \delta\right) \delta+\alpha|\nabla \delta|^{2} \delta+\delta \wedge\left(\Delta \delta+h_{\mathrm{d}}(\delta)\right) \\
& -\alpha \delta \wedge\left(\delta \wedge\left(h_{\mathrm{d}}\left(m_{\mathrm{eq}}(t)\right)+h_{\mathrm{ext}}(t)\right)\right)-\alpha \delta \wedge\left(m_{\mathrm{eq}}(t) \wedge h_{\mathrm{d}}(\delta)\right) \\
& -\alpha m_{\mathrm{eq}}(t) \wedge\left(\delta \wedge h_{\mathrm{d}}(\delta)\right)-\alpha \delta \wedge\left(\delta \wedge h_{\mathrm{d}}(\delta)\right) .
\end{aligned}
$$

In the sequel, we consider

$$
\delta^{\varepsilon}:=m^{\varepsilon}-m_{\mathrm{eq}} \in C\left(\left[0, t^{\varepsilon}\right], H^{2}(\Omega)\right) \cap L^{2}\left(\left(0, t^{\varepsilon}\right), H^{3}(\Omega)\right),
$$


and we simply prove that in $\left(H^{2}\right)$ energy estimates, the term due to the residual $\mathcal{R}\left(m_{\mathrm{eq}}\right) \delta^{\varepsilon}$ is dominated by the terms due to $\alpha \Delta \delta^{\varepsilon}$ and to the linear term $\mathcal{L}\left(m_{\mathrm{eq}}\right) \delta^{\varepsilon}$. We thus come back to the Galerkine approximation $\delta_{k}^{\varepsilon}$ of $\delta^{\varepsilon}$, as in Paragraph 4.1.1. Take the $L^{2}(\Omega)$ scalar product of the equations with $\delta_{k}^{\varepsilon}$ and $\Delta^{2} \delta_{k}^{\varepsilon}$ and integrate by parts. Estimating $\left(\delta_{k}^{\varepsilon} \mid \mathcal{R}\left(m_{\mathrm{eq}}\right)\left(\delta_{k}^{\varepsilon}\right)\right)_{L^{2}(\Omega)}$ is straightforward. Due to the continuity properties of $h_{\mathrm{d}}$ on Sobolev spaces, $\left(\Delta^{2} \delta_{k}^{\varepsilon} \mid \mathcal{R}\left(m_{\mathrm{eq}}\right)\left(\delta_{k}^{\varepsilon}\right)\right)_{L^{2}(\Omega)}$ produces three kinds of terms. Dropping the exponent $\varepsilon$ and subscript $k$ (and using the notation $L\left(v_{1}, \ldots, v_{n}\right)$ for any $n$-linear application), we examine each of them.

From $\delta \wedge\left(\delta \wedge h_{\mathrm{d}}(\delta)\right)$. We have

$\left(\Delta^{2} \delta \mid L(\delta, \delta, \delta)\right)_{L^{2}(\Omega)}=(\Delta \delta \mid \Delta L(\delta, \delta, \delta))_{L^{2}(\Omega)} \leqslant\|\Delta \delta\|_{L^{2}(\Omega)}\|\Delta L(\delta, \delta, \delta)\|_{L^{2}(\Omega)}$,

which is bounded from above by $C\|\delta\|_{H^{2}(\Omega)}^{4}$, since $H^{2}(\Omega)$ is an algebra.

In the same way, the terms of the form $\left(\Delta^{2} \delta \mid L(\delta, \delta)\right)_{L^{2}(\Omega)}$ are controlled by $\|\delta\|_{H^{2}(\Omega)}^{3}$. This rules out the terms from $\delta \wedge h_{\mathrm{d}}(\delta), \delta \wedge\left(\delta \wedge\left(h_{\mathrm{d}}\left(m_{\mathrm{eq}}(t)\right)+h_{\mathrm{ext}}(t)\right)\right)$, $\delta \wedge\left(m_{\mathrm{eq}}(t) \wedge h_{\mathrm{d}}(\delta)\right)$ and $m_{\mathrm{eq}}(t) \wedge\left(\delta \wedge h_{\mathrm{d}}(\delta)\right)$

From $|\nabla \delta|^{2} \delta$. Write

$$
\begin{aligned}
& \left(\Delta^{2} \delta \mid L(\nabla \delta, \nabla \delta, \delta)\right)_{L^{2}(\Omega)}= \\
& \quad-(\nabla \Delta \delta \mid L(\nabla \delta, \nabla \delta, \nabla \delta))_{L^{2}(\Omega)}-(\nabla \Delta \delta \mid \tilde{L}(\Delta \delta, \nabla \delta, \delta))_{L^{2}(\Omega)} .
\end{aligned}
$$

Then,

$$
\begin{aligned}
\left|(\nabla \Delta \delta \mid L(\nabla \delta, \nabla \delta, \nabla \delta))_{L^{2}(\Omega)}\right| & \leqslant\|\nabla \Delta \delta\|_{L^{2}(\Omega)}\|L(\nabla \delta, \nabla \delta, \nabla \delta)\|_{L^{2}(\Omega)} \\
& \leqslant C\|\nabla \Delta \delta\|_{L^{2}(\Omega)}\|\nabla \delta\|_{L^{6}(\Omega)}^{3},
\end{aligned}
$$

and by Sobolev's inequalities, $\|\nabla \delta\|_{L^{6}(\Omega)}$ is controlled by $\|\delta\|_{H^{2}(\Omega)}$.

Also,

$\left|(\nabla \Delta \delta \mid \tilde{L}(\Delta \delta, \nabla \delta, \delta))_{L^{2}(\Omega)}\right| \leqslant C\|\nabla \Delta \delta\|_{L^{2}(\Omega)}\|\Delta \delta\|_{L^{2}(\Omega)}\|\nabla \delta\|_{L^{\infty}(\Omega)}\|\delta\|_{L^{\infty}(\Omega)}$.

¿From the estimate

$$
\|\nabla \delta\|_{L^{\infty}(\Omega)} \lesssim\|\nabla \delta\|_{L^{2}(\Omega)}+\|\nabla \Delta \delta\|_{L^{2}(\Omega)},
$$

we get

$\left|(\nabla \Delta \delta \mid \tilde{L}(\Delta \delta, \nabla \delta, \delta))_{L^{2}(\Omega)}\right| \leqslant C\left(\|\nabla \Delta \delta\|_{L^{2}(\Omega)}\|\delta\|_{H^{2}(\Omega)}^{3}+\|\nabla \Delta \delta\|_{L^{2}(\Omega)}^{2}\|\delta\|_{H^{2}(\Omega)}^{2}\right)$.

This leads to

$$
\left(\Delta^{2} \delta \mid L(\nabla \delta, \nabla \delta, \delta)\right)_{L^{2}(\Omega)} \leqslant C\left(\|\nabla \Delta \delta\|_{L^{2}(\Omega)}\|\delta\|_{H^{2}(\Omega)}^{3}+\|\nabla \Delta \delta\|_{L^{2}(\Omega)}^{2}\|\delta\|_{H^{2}(\Omega)}^{2}\right) .
$$

In the same way, we have

$$
\left(\Delta^{2} \delta \mid\left(\nabla m_{\mathrm{eq}}(t) \cdot \nabla \delta\right) \delta\right) \leqslant C\|\nabla \Delta \delta\|_{L^{2}(\Omega)}\|\delta\|_{H^{2}(\Omega)}^{2}
$$


The $\delta \wedge \Delta \delta$ term. Again,

$$
\left(\Delta^{2} \delta \mid L(\delta, \Delta \delta)\right)_{L^{2}(\Omega)}=-(\nabla \Delta \delta \mid L(\nabla \delta, \Delta \delta))_{L^{2}(\Omega)}-(\nabla \Delta \delta \mid L(\delta, \nabla \Delta \delta))_{L^{2}(\Omega)},
$$

and as above, we get

$$
\left(\Delta^{2} \delta \mid L(\delta, \Delta \delta)\right)_{L^{2}(\Omega)} \leqslant C\left(\|\nabla \Delta \delta\|_{L^{2}(\Omega)}\|\delta\|_{H^{2}(\Omega)}^{2}+\|\nabla \Delta \delta\|_{L^{2}(\Omega)}^{2}\|\delta\|_{H^{2}(\Omega)}\right) .
$$

Finally, there is $C>0$, and for all $\eta>0$, there is $C_{\eta}>0$ such that

$$
\begin{aligned}
\left(\delta_{k}^{\varepsilon} \mid \mathcal{R}\left(m_{\mathrm{eq}}\right)\left(\delta_{k}^{\varepsilon}\right)\right)_{H^{2}(\Omega)} \leqslant & \left(\eta+C\left\|\delta_{k}^{\varepsilon}\right\|_{H^{2}(\Omega)}+C\left\|\delta_{k}^{\varepsilon}\right\|_{H^{2}(\Omega)}^{2}\right)\left\|\nabla \Delta \delta_{k}^{\varepsilon}\right\|_{L^{2}(\Omega)}^{2} \\
& +C_{\eta}\left(\left\|\delta_{k}^{\varepsilon}\right\|_{H^{2}(\Omega)}^{3}+\left\|\delta_{k}^{\varepsilon}\right\|_{H^{2}(\Omega)}^{4}+\left\|\delta_{k}^{\varepsilon}\right\|_{H^{2}(\Omega)}^{6}\right) .
\end{aligned}
$$

Let $k$ go to infinity, so that the above estimate applies to $\delta^{\varepsilon}$ instead of $\delta_{k}^{\varepsilon}$, up to the local existence time $t^{\varepsilon}$ obtained via the convergence of the Galerkine scheme. Coming back to (4.29), still with $\delta^{\varepsilon}=m^{\varepsilon}-m_{\text {eq }}$, we get, using (2.5): there is $C>0$, and for all $\eta>0$, there is $C_{\eta}>0$ such that

$$
\begin{aligned}
& \frac{\varepsilon}{2} \frac{\mathrm{d}}{\mathrm{dt}}\left(\left\|\delta^{\varepsilon}\right\|_{H^{2}(\Omega)}^{2}\right)+\left(\alpha-\eta-C\left(1+\left\|\delta^{\varepsilon}\right\|_{H^{2}(\Omega)}\right)\left\|\delta^{\varepsilon}\right\|_{H^{2}(\Omega)}\right)\left\|\nabla \delta^{\varepsilon}\right\|_{H^{2}(\Omega)}^{2} \\
& \quad \leqslant\left(C_{\eta}\left(1+\left\|\delta^{\varepsilon}\right\|_{H^{2}(\Omega)}\right)^{3}\left\|\delta^{\varepsilon}\right\|_{H^{2}(\Omega)}-C_{\mathrm{lin}}\right)\left\|\delta^{\varepsilon}\right\|_{H^{2}(\Omega)}^{2}+\varepsilon^{2}\left\|\partial_{t} m_{\mathrm{eq}}\right\|_{H^{2}(\Omega)}^{2} .
\end{aligned}
$$

As in the proof of Lemma 4.2, fix $\eta \in(0, \alpha)$, and consider the time $\tilde{t^{\varepsilon}} \leqslant t^{\varepsilon}$ up to which, in (4.33), the parenthesis in front of $\left\|\nabla \delta^{\varepsilon}\right\|_{H^{2}(\Omega)}^{2}\left(\right.$ resp. $\left.\left\|\delta^{\varepsilon}\right\|_{H^{2}(\Omega)}^{2}\right)$ remains positive (resp. less than $-C_{\text {lin }} / 2$ ). We have, for $t \in\left(t_{\varepsilon}, \tilde{t^{\varepsilon}}\right)$ :

$$
\frac{\varepsilon}{2} \frac{\mathrm{d}}{\mathrm{dt}}\left(\left\|\delta^{\varepsilon}\right\|_{H^{2}(\Omega)}^{2}\right) \leqslant-\frac{C_{\text {lin }}}{2}\left\|\delta^{\varepsilon}\right\|_{H^{2}(\Omega)}^{2}+\varepsilon^{2}\left\|\partial_{t} m_{\mathrm{eq}}\right\|_{H^{2}(\Omega)}^{2} .
$$

Gronwall's lemma then implies that

$$
\sup _{\left[t_{\varepsilon}, t^{\tilde{t}}\right]}\left\|\delta^{\varepsilon}\right\|_{H^{2}(\Omega)}^{2} \leqslant\left\|\delta^{\varepsilon}\left(t_{\varepsilon}\right)\right\|_{H^{2}(\Omega)}^{2}+2 \varepsilon T \sup _{[0, T]}\left\|\partial_{t} m_{\mathrm{eq}}\right\|_{H^{2}(\Omega)}^{2}
$$

so that, for $\varepsilon$ small enough, we get $\tilde{t^{\varepsilon}} \geqslant T$, and $\sup _{\left[t_{\varepsilon}, T\right]}\left\|\delta^{\varepsilon}\right\|_{H^{2}(\Omega)} \underset{\varepsilon \rightarrow 0}{\longrightarrow} 0$. This finishes the proof of Theorem 2.1.

\section{Proof of Proposition 2.2 and Corollary 2.3}

Proof of Proposition 2.2. For any $T>0$ and $n \in C\left([0, T], H^{2}(\Omega)\right)$, it is equivalent for $n$ to be solution to (2.8) or to

$$
\left(\partial_{t}-\alpha \Delta\right)\left(n-m_{\mathrm{eq}}\right)=\left(\mathcal{L}\left(0, m_{\mathrm{eq}}\right)+\mathcal{R}\left(0, m_{\mathrm{eq}}\right)\right)\left(n-m_{\mathrm{eq}}\right),
$$


with the same initial and boundary conditions. The operators $\mathcal{L}\left(0, m_{\mathrm{eq}}\right)$ and $\mathcal{R}\left(0, m_{\mathrm{eq}}\right)$ from (4.31) and (4.32) do not depend on time, now. Arguing as in Section 4.2, we get an estimate analogue to (4.33),

$$
\begin{aligned}
& \frac{1}{2} \frac{\mathrm{d}}{\mathrm{dt}}\left(\left\|n-m_{\mathrm{eq}}\right\|_{H^{2}(\Omega)}^{2}\right) \\
& \quad+\left(\alpha-\eta-C\left(1+\left\|n-m_{\mathrm{eq}}\right\|_{H^{2}(\Omega)}\right)\left\|n-m_{\mathrm{eq}}\right\|_{H^{2}(\Omega)}\right)\left\|\nabla\left(n-m_{\mathrm{eq}}\right)\right\|_{H^{2}(\Omega)}^{2} \\
& \quad \leqslant\left(C_{\eta}\left(1+\left\|n-m_{\mathrm{eq}}\right\|_{H^{2}(\Omega)}\right)^{3}\left\|n-m_{\mathrm{eq}}\right\|_{H^{2}(\Omega)}-C_{\mathrm{lin}}\right)\left\|n-m_{\mathrm{eq}}\right\|_{H^{2}(\Omega)}^{2} .
\end{aligned}
$$

Once $\eta \in(0, \alpha / 2)$ is chosen, take $\eta_{0}>0$ such that, when $\left\|m_{0}-m_{\mathrm{eq}}\right\|_{H^{2}(\Omega)} \leqslant \eta_{0}$, the parentheses in front of $\left\|\nabla\left(n-m_{\mathrm{eq}}\right)\right\|_{H^{2}(\Omega)}^{2}$ and in front of $\left\|n-m_{\mathrm{eq}}\right\|_{H^{2}(\Omega)}^{2}$ are positive and negative at $t=0$, respectively. The bootstrap argument then shows that $n \in C\left([0, \infty), H^{2}(\Omega)\right)$, and that $n(t)$ converges in $H^{2}\left(\Omega, S^{2}\right)$, as $t$ goes to $\infty$, towards $m_{\text {eq }}\left(t_{0}\right)$ :

$$
\left\|n(t)-m_{\mathrm{eq}}\right\|_{H^{2}(\Omega)} \leq \eta_{0} e^{-C t},
$$

for some $C \in\left(0, C_{\text {lin }}\right)$ depending on $\eta_{0}$. Coming back to (5.1), we see also that $\nabla\left(n-m_{\mathrm{eq}}\right) \in L^{2}\left((0, \infty), H^{2}(\Omega)\right)$.

Proof of Corollary 2.3. When $m_{\mathrm{eq}}(0)$ is constant over $\Omega$, Proposition 2.2 ensures there exists some $\eta_{0}>0$ such that for all $m_{0} \in H_{N}^{2}\left(\Omega, S^{2}\right)$ satisfying

$$
\left\|m_{0}-m_{\mathrm{eq}}\right\|_{H^{2}(\Omega)} \leqslant \eta_{0},
$$

Asumption (ii) in Theorem 2.1 holds true. Furthermore, estimations (5.1) and (5.2) show that the corresponding function $n_{0}$ has norms in $L^{\infty}\left((0, \infty), H^{2}(\Omega)\right)$ and in $L^{2}((0, \infty) \times \Omega)$ ) controlled in terms of $m_{\mathrm{eq}}(0)$ and $\eta_{0}$ only. Thus, $\varepsilon_{0}$ in the proof of Theorem 2.1 may also be chosen depending on $m_{\text {eq }}(0)$ and $\eta_{0}$ only, uniformly with respect to $m_{0}$.

\section{Appendix}

\subsection{About the dissipation property (2.5): proof of Lem- mas 3.5 and 3.6}

Let $\delta \in C\left([0, T], H^{\infty}(\Omega)\right)$ be such that $\left|m_{\mathrm{eq}}+\delta\right| \equiv 1$ and $\partial_{\nu} \delta_{\mid \partial \Omega}=\partial_{\nu} \Delta \delta_{\mid \partial \Omega}=0$. Then,

(6.1)

$\mathcal{L}\left(m_{\mathrm{eq}}^{ \pm}\right) \delta=(\lambda \mp d) \delta \wedge u \pm u \wedge\left(\Delta \delta+h_{\mathrm{d}}(\delta)\right)+\alpha(d \mp \lambda) u \wedge(\delta \wedge u)-\alpha u \wedge\left(u \wedge h_{\mathrm{d}}(\delta)\right)$. 


\subsection{1 $\quad L^{2}$ estimates}

Take the $L^{2}(\Omega)$ scalar product of (6.1) with $\delta$. This yields

$$
\begin{aligned}
\left(\mathcal{L}\left(m_{\mathrm{eq}}^{ \pm}\right) \delta \mid \delta\right)_{L^{2}(\Omega)} & = \pm \int_{\Omega} \delta \cdot(u \wedge \Delta \delta) \pm \int_{\Omega} \delta \cdot\left(u \wedge h_{\mathrm{d}}(\delta)\right) \\
& +\alpha(d \mp \lambda) \int_{\Omega}|\delta \wedge u|^{2}-\alpha \int_{\Omega}\left(u \cdot h_{\mathrm{d}}(\delta)\right)(u \cdot \delta)+\alpha \int_{\Omega} \delta \cdot h_{\mathrm{d}}(\delta) .
\end{aligned}
$$

First consider the case of $m_{\mathrm{eq}}^{+}$. Denoting $n$ the exterior normal vector to $\Omega$, the first term in the right-hand side of (6.2) is equal to

$$
\sum_{i=1}^{3} \int_{\Omega} \delta \cdot \partial_{i}\left(u \wedge \partial_{i} \delta\right)=\sum_{i=1}^{3} \int_{\partial \Omega} \delta \cdot\left(u \wedge \partial_{i} \delta\right) n_{i}=\int_{\partial \Omega} \delta \cdot\left(u \wedge \partial_{n} \delta\right)=0
$$

Since $h_{\mathrm{d}}$ is continuous on $L^{2}$ with norm 1 , the second term is bounded from above by $\|\delta\|_{L^{2}(\Omega)}^{2}$. Similarly, due to the non-positivity of $h_{\mathrm{d}}$, the last term is non-positive. In the two other terms, we inject the identities

$$
|\delta|^{2}=-2 u \cdot \delta \quad \text { and } \quad|\delta \wedge u|^{2}=|\delta|^{2}-\frac{1}{4}|\delta|^{4},
$$

which stem from the equality $|u+\delta| \equiv 1$. This leads to

$$
\begin{array}{r}
\left(\mathcal{L}\left(m_{\mathrm{eq}}^{+}\right) \delta \mid \delta\right)_{L^{2}(\Omega)} \leqslant\|\delta\|_{L^{2}(\Omega)}^{2}+\alpha(d-\lambda) \int_{\Omega}\left(|\delta|^{2}-|\delta|^{4} / 4\right) \\
+\frac{\alpha}{2} \int_{\Omega}\left(u \cdot h_{\mathrm{d}}(\delta)\right)|\delta|^{2} \\
=(1+\alpha(d-\lambda))\|\delta\|_{L^{2}(\Omega)}^{2}+\mathcal{O}\left(\|\delta\|_{L^{2}(\Omega)}^{3}\right) .
\end{array}
$$

In the case of $m_{\text {eq }}^{-}$, we obtain in the same way

$$
\left(\mathcal{L}\left(m_{\mathrm{eq}}^{-}\right) \delta \mid \delta\right)_{L^{2}(\Omega)} \geqslant(\alpha(\lambda+d)-c)\|\delta\|_{L^{2}(\Omega)}^{2}+\mathcal{O}\left(\|\delta\|_{L^{2}(\Omega)}^{3}\right),
$$

for some constant $c$ depending on $\Omega$ and $\alpha$ only.

\subsection{2 $H^{2}$ estimates}

Take the $L^{2}(\Omega)$ scalar product of the Laplacian of each term in (6.1) with $\Delta \delta$. This yields

$$
\begin{aligned}
\left(\Delta \mathcal{L}\left(m_{\mathrm{eq}}^{ \pm}\right) \delta \mid \Delta \delta\right)_{L^{2}(\Omega)}= & (\lambda \mp d) \int_{\Omega} \Delta \delta \cdot \Delta(\delta \wedge u) \pm \int_{\Omega} \Delta \delta \cdot \Delta(u \wedge \Delta \delta) \\
& \pm \int_{\Omega} \delta \cdot \Delta\left(u \wedge h_{\mathrm{d}}(\delta)\right)+\alpha(d \mp \lambda) \int_{\Omega}|\Delta \delta \wedge u|^{2} \\
& -\alpha \int_{\Omega} \Delta \delta \cdot \Delta\left(u \wedge\left(u \wedge h_{\mathrm{d}}(\delta)\right)\right) .
\end{aligned}
$$


Since $\Delta(\delta \wedge u)=(\Delta \delta) \wedge u=0$, the first term on the right-hand side vanishes. So does the second one, by the same argument as in (6.3). The equality $|u+\delta| \equiv 1$ implies

$$
|\Delta \delta \wedge u|^{2}=|\Delta \delta|^{2}-\left(|\nabla \delta|^{2}-(\delta \cdot \Delta \delta)^{2}\right)^{2},
$$

so that (6.7) gives, for $m_{\mathrm{eq}}^{+}$:

$$
\left(\Delta \mathcal{L}\left(m_{\mathrm{eq}}^{+}\right) \delta \mid \Delta \delta\right)_{L^{2}(\Omega)} \leqslant-\alpha(\lambda-d)\|\Delta \delta\|_{L^{2}(\Omega)}^{2}+c\|\delta\|_{H^{2}(\Omega)}^{2}+\mathcal{O}\left(\|\delta\|_{H^{2}(\Omega)}^{3}\right),
$$

for some constant $c$ depending on $\Omega$ and $\alpha$ only. Together with (6.5), we get finally

$$
\left(\mathcal{L}\left(m_{\mathrm{eq}}^{+}\right) \delta \mid \delta\right)_{H^{2}(\Omega)} \leqslant-(\alpha(\lambda-d)-c)\|\delta\|_{H^{2}(\Omega)}^{2}+\mathcal{O}\left(\|\delta\|_{H^{2}(\Omega)}^{3}\right),
$$

which concludes the proof of Lemma 3.5.

In the case of $m_{\text {eq }}^{-}$, we have

$$
\begin{aligned}
\left(\Delta \mathcal{L}\left(m_{\mathrm{eq}}^{+}\right) \delta \mid \Delta \delta\right)_{L^{2}(\Omega)}= & -\int_{\Omega} \Delta \delta \cdot \Delta\left(u \wedge h_{\mathrm{d}}(\delta)\right)+\alpha(\lambda+d)\|\Delta \delta\|_{L^{2}(\Omega)}^{2} \\
& -\alpha \int_{\Omega} \Delta \delta \cdot \Delta\left(u \wedge\left(u \wedge h_{\mathrm{d}}(\delta)\right)\right)+\mathcal{O}\left(\|\delta\|_{H^{2}(\Omega)}^{3}\right),
\end{aligned}
$$

which, together with (6.6), leads to Lemma 3.6.

\subsection{Proof of the commutator lemma 4.1}

Writting

$$
\left[P_{k}, \mathcal{F}(0, \cdot)\right](n)=\left(P_{k}-1\right) \mathcal{F}(0, n)+\mathcal{F}(0, n)-\mathcal{F}\left(0, P_{k} n\right),
$$

the result follows from the convergence of $P_{k}$ towards 1 pointwise as an operator on $H^{1}(\Omega)$ (which rules out the term $\left(P_{k}-1\right) \mathcal{F}(0, n)$ ) as well as on $H_{N}^{2}(\Omega)$, combined (to deal with $\mathcal{F}(0, n)-\mathcal{F}\left(0, P_{k} n\right)$ ) with the continuity of $\mathcal{F}(0, \cdot)$ from $C\left([0, T], H^{2}(\Omega)\right) \cap L^{2}\left((0, T), H^{3}\right)$ to $L^{2}\left((0, T), H^{1}\right)$.

The latter is a consequence of the continuity properties of $h_{\mathrm{d}}$ and of Sobolev's embeddings, implying that $H^{2}(\Omega)$ is an algebra (so that all applications $n \mapsto$ $n \wedge h_{\mathrm{d}}(n), n \mapsto n \wedge\left(n \wedge h_{\mathrm{d}}(n)\right), n \mapsto n \wedge h_{\text {ext }}(0), n \mapsto n \wedge\left(n \wedge h_{\text {ext }}(0)\right)$ are continuous on $\left.L^{\infty}\left((0, T), H^{2}(\Omega)\right)\right)$, and that the product operation maps $H^{2} \times H^{1}$ to $H^{1}$, so that $n \mapsto n \wedge \Delta n$ and $n \mapsto|\nabla n|^{2} n$ are continuous from $L^{\infty}\left((0, T), H^{2}(\Omega)\right) \cap L^{2}\left((0, T), H^{3}\right)$ to $L^{2}\left((0, T), H^{1}\right)$.

\subsection{Proof of Gronwall's lemma 4.2}

First, consider $k \in \mathbb{N}^{\star}$ and $\varepsilon>0$ fixed. Set $\phi^{\varepsilon}(t)=\left\|\varphi_{k}^{\varepsilon}\right\|_{H^{2}(\Omega)}^{2}, r(t)=\tilde{r}_{k}^{\varepsilon}(t)$, $N_{0}(t)=\left\|\nabla \Delta P_{k} n_{0}(t)\right\|_{L^{2}(\Omega)}^{2}$ and $N^{\varepsilon}(t)=\left\|\nabla \Delta n_{k}^{\varepsilon}(t)\right\|_{L^{2}(\Omega)}^{2}$, so that

$$
N^{\varepsilon}(t)=N_{0}(t / \varepsilon) \quad \text { and } \quad N_{0} \in L^{1}(0, \infty) .
$$


With $C$ from (4.27), choose $\eta \in(0, \alpha /(2 C))$. Hence, there exists $\kappa_{\eta} \in(0,1)$ such that

$$
\forall \varphi \in\left[0, \kappa_{\eta}\right], \quad C(\eta+\varphi(1+\varphi))<\alpha / 2 .
$$

Set $K=8 C_{\eta}$ (also from (4.27)), $c \in(0,1 / K)$ and $t_{\varepsilon}=c \varepsilon \ln (1 / \varepsilon)$. Then, with

$$
t_{k}^{\varepsilon}=\sup \left\{t \in\left[0, t_{\varepsilon}\right] \mid \forall t^{\prime} \in[0, t], \phi^{\varepsilon}\left(t^{\prime}\right) \leqslant \kappa_{\eta}\right\}
$$

$\left(t_{k}^{\varepsilon}>0\right.$ since $\left.\phi^{\varepsilon}(0)=0\right)$, we have:

$$
\forall t \in\left[0, t_{k}^{\varepsilon}\right], \quad \varepsilon \phi^{\varepsilon^{\prime}}(t) \leqslant K\left(\left(1+N^{\varepsilon}(t)\right) \phi^{\varepsilon}(t)+t+r(t)\right) .
$$

¿From this, we deduce:

$$
\begin{aligned}
\forall t \in\left[0, t_{k}^{\varepsilon}\right], \quad \phi^{\varepsilon}(t) & \leqslant \int_{0}^{t} \frac{K}{\varepsilon}\left(t^{\prime}+r\left(t^{\prime}\right)\right) \exp \left(\frac{K}{\varepsilon} \int_{t^{\prime}}^{t}\left(1+N^{\varepsilon}\left(t^{\prime \prime}\right)\right) d t^{\prime \prime}\right) d t^{\prime} \\
& \leqslant\left(\int_{0}^{t} \frac{K}{\varepsilon}\left(t^{\prime}+r\left(t^{\prime}\right)\right) \exp \left(K \frac{t-t^{\prime}}{\varepsilon}\right) d t^{\prime}\right) e^{K\left\|N_{0}\right\|_{L^{1}(0, \infty)}} \\
& \leqslant\left(\frac{\varepsilon}{K}+\frac{K}{\varepsilon}\|r\|_{L^{1}\left(0, T_{0}\right)}\right) e^{K t / \varepsilon} e^{K\left\|N_{0}\right\|_{L^{1}(0, \infty)}},
\end{aligned}
$$

with $T_{0}=c \varepsilon_{0} \ln \left(1 / \varepsilon_{0}\right)$ (and $\varepsilon_{0}$ is chosen below). Now, since $c \in(0,1 / K)$ and $t_{\varepsilon}=c \varepsilon \ln (1 / \varepsilon)$,

$$
\forall t \in\left[0, \min \left(t_{k}^{\varepsilon}, t_{\varepsilon}\right)\right], \quad \phi^{\varepsilon}(t) \leqslant\left(\frac{\varepsilon^{1-c K}}{K}+K\|r\|_{L^{1}\left(0, T_{0}\right)} \varepsilon^{-1-c K}\right) e^{K\left\|N_{0}\right\|_{L^{1}(0, \infty)}},
$$

which is less or equal to $\kappa_{\eta}$ as soon as $\varepsilon$ belongs to $\left(0, \varepsilon_{0}\right]$, for

$$
\varepsilon_{0}=\left(\frac{1}{2} \kappa_{\eta} K e^{-K\left\|N_{0}\right\|_{L^{1}(0, \infty)}}\right)^{1 /(1-c K)}
$$

and $k$ greater than $K(\varepsilon)$ such that

$$
\forall k \geqslant K(\varepsilon), \quad\left\|\tilde{r}_{k}^{\varepsilon}\right\|_{L^{1}\left(0, T_{0}\right)} \leqslant \frac{1}{2} \kappa_{\eta} \frac{\varepsilon^{1+c K}}{K} e^{-K\left\|N_{0}\right\|_{L^{1}(0, \infty)}}
$$

(which is possible by Lemma 4.1). For this choice of $\varepsilon$ and $k$, we thus have $t_{k}^{\varepsilon} \geqslant t_{\varepsilon}$, and the result follows.

\section{References}

[1] F. Alouges and K. Beauchard. Magnetization switching on small ferromagnetic ellipsoidal samples. ESAIM: COCV, 15:676-711, 2009.

[2] J.-P. Aubin. Un théorème de compacité. C. R. Acad. Sci. Paris, 256:5042$5044,1963$. 
[3] G. Carbou, M. Effendiev, and P. Fabrie. Relaxed model for the hysteresis in micromagnetism. Preprint, 2009.

[4] G. Carbou and P. Fabrie. Regular solutions for landau-lifshitz equation in a bounded domain. Differential and integral equations, 14:219-229, 2001.

[5] Robert Dautray and Jacques-Louis Lions. Analyse mathématique et calcul numérique pour les sciences et les techniques. Tome 2. Collection du Commissariat à l'Énergie Atomique: Série Scientifique. [Collection of the Atomic Energy Commission: Science Series]. Masson, Paris, 1985. With the collaboration of Michel Artola, Philippe Bénilan, Michel Bernadou, Michel Cessenat, Jean-Claude Nédélec, Jacques Planchard and Bruno Scheurer.

[6] F. Diener and G. Reeb. Analyse non standard. Hermann, Paris, 1989.

[7] L. Landau and E. Lifshitz. Électrodynamique des milieux continus. Cours de physique théorique, volume 8. Mir ed., Moscou, 1969.

[8] J.A. Osborn. Demagnetizing factors of the general ellipsoid. Phys. Rev., 67:351-357, 1945.

[9] Lord Rayleigh. On the behaviour of iron and steel under the operation of feeble magnetic forces. Phil. Mag., 1887.

[10] J. Simon. Compact sets in the space $L^{p}(0, T ; B)$. Ann. Mat. Pura Appl. (4), 146:65-96, 1987.

[11] J. Starynkevitch. Problèmes dasymptotique en temps en ferromagnétisme. PhD Thesis, 2006.

[12] A. Visintin. Differential Models of Hysteresis, volume 111 of Applied Mathematical Science. Springer Verlag, 1994. 